\title{
Die Virtuelle Bibliothek der Max-Planck-Gesellschaft / Max Planck Virtual Library: Konzeption und Perspektiven'
}

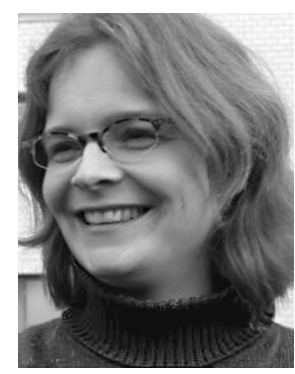

Mit der Virtuellen Bibliothek steht den Wissenschaftlern der Max-Planck-Gesellschaft ein Portal zur Verfügung, das einen Überblick über die ihnen verfügbaren Informationsressourcen sowie eine parallele Suchmöglichkeit in diesen Ressourcen bietet. Ein kontextsensitives Linksystem ermöglicht, ausgehend von den Suchergebnissen, Zugang zu weiteren relevanten Angeboten, z. B. Verfügbarkeitsinformationen. In dem Artikel wird die Entstehung der Virtuellen Bibliothek der MPG dargestellt und das Serviceangebot dieses Portals beschrieben.

The Max Planck Virtual Library

The Virtual Library is a portal for the researchers in the Max Planck Society, which gives them an overview of the information resources available to them and makes it possible to search these resources simultaneously. A context sensitive linking system provides access from the search results to further relevant services. This article describes the implementation of the Max Planck Virtual Library and its services.

La bibliothèque virtuelle de la Société Max Planck

La bibliothèque virtuelle constitue pour les chercheurs de la Société Max Planck un portail qui leur donne un aperçu sur les ressources d'informations mises à leur disposition et qui, simultanément, leur permet d'entreprendre des recherches à l'intérieur de ces ressources. Un système de liens qui prend en compte le contexte de la recherche leur donne, au-delà des résultats, l'accès à des informations supplémentaires. Cet article traite de la naissance de la bibliothèque virtuelle de la Société Max Planck et décrit les services proposés par ce portail. 


\section{Inhaltsverzeichnis}

1 Einleitung.

2 Das Umfeld

2.1 Elektronische Ressourcen und Zugangssysteme im wissenschaftlichen Informationswesen .................... 231

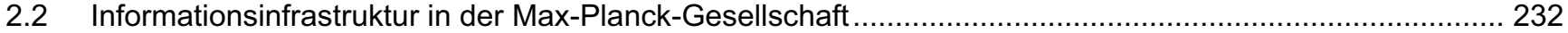

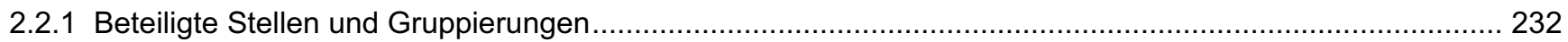

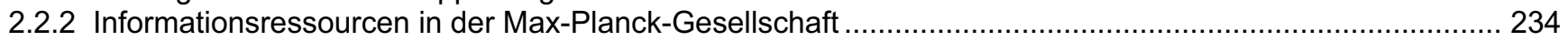

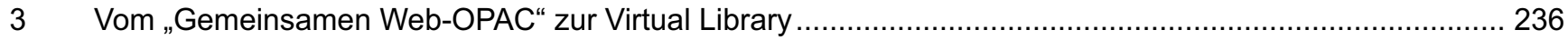

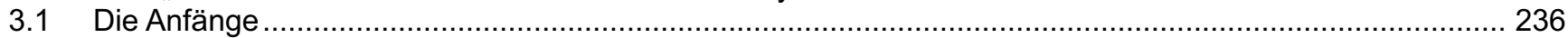

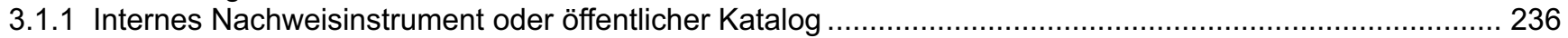

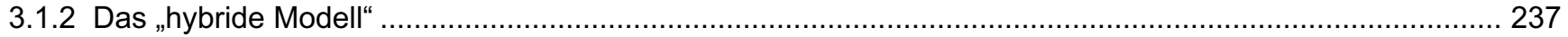

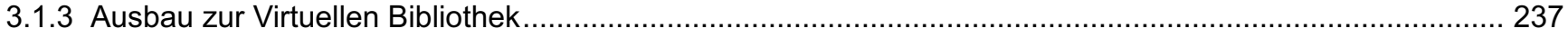

3.2 Produktauswahl für die Max Planck Virtual Library ........................................................................ 238

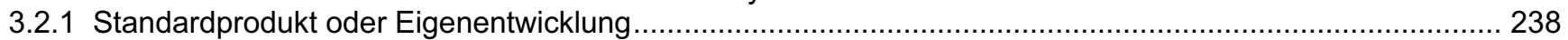

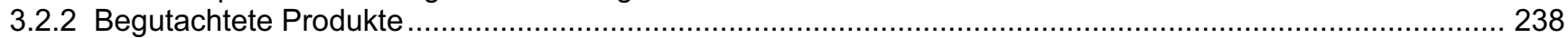

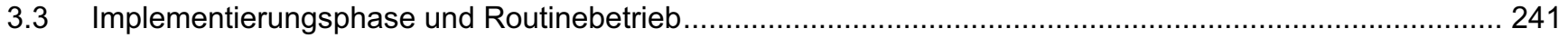

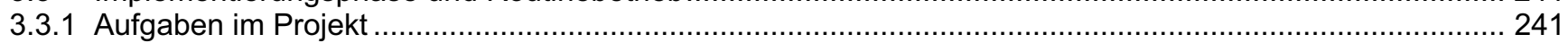

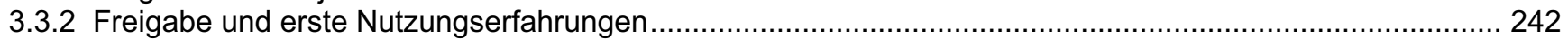

$4 \quad$ Das Serviceangebot der Max Planck Virtual Library ............................................................................ 243

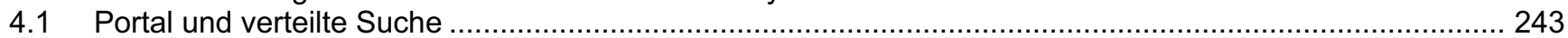

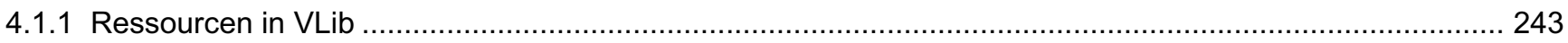

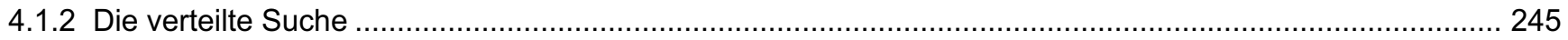

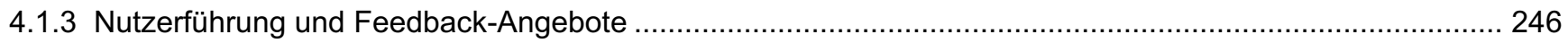

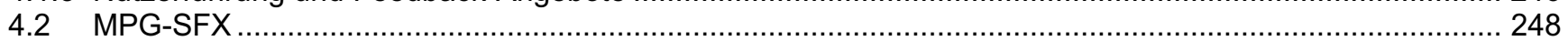

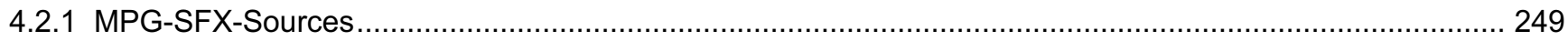

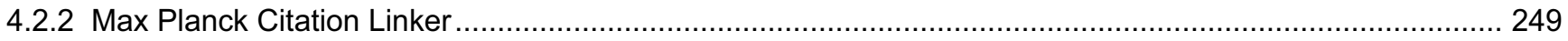

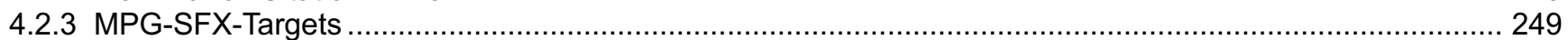

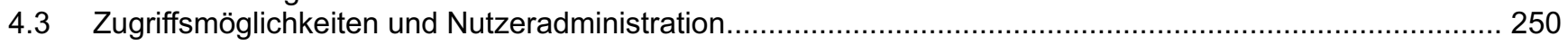

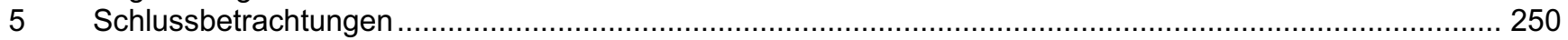

\section{$1 \quad$ Einleitung}

Die Max Planck Virtual Library² (Max Planck VLib) ist ein wissenschaftliches Informationsportal, das sich an die Wissenschaftlerinnen und Wissenschaftler an den Instituten, Forschungsstellen und Projektgruppen in der Max-PlanckGesellschaft wendet und innen einen integrierten Zugang zu zahlreichen elektronischen Informationsressourcen anbietet. Die Motivation beim Aufbau dieses Portals war die Schaffung eines ",bruchlosen Informationsraums“" ${ }^{\text {" }}$, in dem internetbasierte Quellen für die Benutzer komfortabel erschlossen und leicht auffindbar sind: „Mittelfristiges Ziel ist der Ausbau des Systems zu einer entwickelten hybriden virtuellen Bibliothek der Max-Planck-Gesellschaft, die integrierten Zugang zu allen relevanten Informationsressourcen mit maximaler Personalisierung und der Selbstverständlichkeit des Zugriffs von überall auf alles bietet"4.

Um die Nutzbarkeit eines solchen Systems in einem fachlich, aber auch im Hinblick auf die Organisation des Informationsmanagements der Einrichtungen sehr heterogenen Umfeld sicherzustellen, müssen die angebotenen Quellen dabei durch lokalisierte oder auch personalisierte Sichten fachspezifisch auswählbar sein und der Zugriff auf sie gegebenenfalls auf berechtigte Benutzerkreise eingeschränkt werden können.

Die Max Planck Virtual Library ist seit Oktober 2002 für die Benutzung freigeschaltet, so dass bereits einige Erfahrungen, Rückmeldungen und Nutzungsstatistiken vorliegen. Mit dem Umstieg auf eine neue Version der verwendeten Software im August 2003 konnten einige zum Teil wesentliche Funktionen und Services zur Verfügung ge- stellt werden, die anfänglich noch nicht implementiert waren und die als wesentlich für eine breite Akzeptanz des Angebots durch die Nutzer angesehen werden.

In dieser Arbeit wird die Entstehung der Max Planck Virtual Library vor dem Hintergrund der Informationsinfrastruktur der Max-Planck-Gesellschaft dargestellt; zudem werden die Entscheidungsprozesse aufgezeichnet, die der Durchführung dieses Projekts zugrunde lagen. Es werden ferner einige Aspekte der technischen Realisierung und die für die Umsetzung dieses Vorhabens erforderlichen Arbeitsschritte beschrieben, um die Komplexität eines solchen Projekts deutlich zu machen. Aufbau und Funktionsweise des Informationsportals sowie die darin angebotenen $\mathrm{Da}$ tenquellen und Services werden beschrieben und geplante Weiterentwicklungen aufgezeichnet.

1 Dieser Beitrag ist die überarbeitete und aktualisierte Fassung einer Hausarbeit im Rahmen eines Postgradualen Fernstudiums, die am Institut für Bibliothekswissenschaft der Humboldt-Universität Berlin im Frühjahr 2003 angefertigt wurde.

$2<$ http://vlib.mpg.de>.

${ }^{3}$ Schimmer, Ralf: Auf dem Weg zu einem bruchlosen Informationsraum. Präsentation zur IUK-Tagung 2002 in Ulm <http:// www.zim.mpg.de/projects/virtlib/iuk-ulm2002.ppt>.

${ }^{4}$ Schimmer (Anm. 3) Folie 21. 
Zum Ausdruck kommen soll einerseits die Rolle, die dieses Portal bei der Kooperation der verschiedenen Einrichtungen in der Max-Planck-Gesellschaft im Bereich des wissenschaftlichen Informationsmanagements spielt, wobei Perspektiven und Weiterentwicklungen im Bereich der Informationsinfrastruktur erkennbar werden sollen. Andererseits soll aber auch deutlich werden, welche Überlegungen und Arbeitsschritte zur Umsetzung eines vergleichbaren Vorhabens notwendig sind und auf welche Probleme Bibliothekare und Informationsvermittler, die sich einer solchen Aufgabe widmen, stoßen können.

Als Quellen für diese Arbeit wurden neben wissenschaftlicher Literatur (gedruckt oder online), Websites und anderen über das WWW zugänglichen Dokumenten insbesondere auch interne Arbeitspapiere, Protokolle, Intranetseiten oder auch E-Mails herangezogen. Grundlage für die Ausführungen bilden ferner Gespräche mit am Projekt beteiligten sowie verantwortlichen Personen, insbesondere Herrn Dr. Schimmer aus dem Sachgebiet Wissenschaftliches Informations-, Dokumentations- und Bibliothekswesen in der Generalverwaltung der Max-Planck-Gesellschaft und Frau Overkamp vom Heinz-Nixdorf-Zentrum für Informationsmanagement in der Max-Planck-Gesellschaft. Etliche Erkenntnisse konnten auch aus der Mitarbeit in der VLib-Projektgruppe gewonnen werden.

\section{Das Umfeld}

Dieses Kapitel beschreibt den Hintergrund, vor dem die Planung und Realisierung der Virtuellen Bibliothek der Max-Planck-Gesellschaft zu sehen ist. Relevante Entwicklungen im Informationswesen werden kurz angesprochen, um dann auf die besonderen Gegebenheiten in der Informationsinfrastruktur der Max-Planck-Gesellschaft einzugehen, die für dieses Projekt von Bedeutung sind.

\subsection{Elektronische Ressourcen und Zugangssyste- me im wissenschaftlichen Informationswesen}

Die Entwicklung des wissenschaftlichen Informationswesens weist einige markante Grundlinien auf, die für die Informationsinfrastruktur in der Max-Planck-Gesellschaft mitbestimmend sind. Als grundlegende Faktoren sind dabei das Vorliegen von elektronisch gespeicherten Informationsmaterialien und ihre webbasierte Verbreitung anzusehen. Es steht außer Frage, dass diese Entwicklungen das wissenschaftliche Informationswesen sowohl im kommerziellen als auch im öffentlich-rechtlichen Bereich nachhaltig verändert haben und sich somit auch auf die im Bereich der Informationsvermittlung tätigen Institutionen und ihre Arbeit auswirken.

Die traditionellen wissenschaftlichen Publikationsformen Monografie, Sammelband oder Zeitschrift werden, besonders in den naturwissenschaftlichen Fächern, nicht mehr dem Tempo der Gewinnung neuer Erkenntnisse und ihrer Bekanntmachung gerecht. Daher haben besonders hier, aber auch in anderen wissenschaftlichen Disziplinen Publikationsformen, die auf elektronischer Datenspeicherung und -übermittlung basieren, an Bedeutung gewonnen. Nicht nur werden Zeitschriftenartikel, Dissertationen oder Bücher - entweder zusätzlich zur Druckversion oder ausschließlich - in elektronischer Form aufgelegt, sondern es spielen auch alternative Formen wissenschaftlichen Informationsaustausches eine Rolle, so etwa die vor der ei- gentlichen Publikation stattfindende Veröffentlichung auf Preprint-Servern, aber auch der informelle Austausch zwischen Wissenschaftlern, etwa in Mailinglisten.

Neben Publikationen und Preprints werden auch andere Materialien in elektronischer Form vorgehalten und verbreitet. Dazu gehören retrodigitalisiertes Material, aber auch Informationen, die in gedruckter Form kaum oder gar nicht vermittelt werden können, z. B. multimediale Quellen, dreidimensionale Bilder und andere visualisierende Darstellungen, Computerprogramme usw. Für das Auffinden von Informationen und Dokumenten stehen eine Vielzahl an Literatur-, Fakten- oder Patentdatenbanken zur Verfügung.

Daneben hat die Bedeutung gedruckter Literatur - obwohl dies im Zusammenhang mit der raschen Vermehrung elektronischer Publikationsformen teils befürchtet, teils erhofft wurde ${ }^{5}$ - bislang nicht in erwartetem Maße abgenommen. Elektronische Kataloge (OPACs), in denen Bibliotheksbenutzer zumeist online und von überall aus nach den Katalogdaten und Statusinformationen über die verfügbare gedruckte Literatur recherchieren können, zählen daher zu den wichtigsten Arbeitsmitteln vieler Wissenschaftler.

Das World Wide Web ermöglicht einen unproblematischen Zugang vom Arbeitsplatz des Wissenschaftlers aus zu vielen dieser verschiedenartigen Informationsmaterialien. Aufgrund der großen Anzahl kostenpflichtiger, aber auch frei zugänglicher Angebote ist es notwendig geworden, Erschließungs- und Zugangssysteme zu schaffen, die die vorhandenen Ressourcen in übersichtlicher Weise präsentieren.

Mit solchen Zugangssystemen werden sowohl die elektronischen Bestände einer einzelnen Institution erschlossen als auch die von ihr lizenzierten Datenbanken oder Zeitschriften sowie diejenigen Informationsangebote, auf die im Rahmen einer Kooperation mehrerer Institutionen, etwa durch Konsortienbildung, ein „Cross Access“ für alle Kooperationspartner gegeben ist. Oft sind auch kostenfreie WWW-Quellen enthalten. Häufig ist, besonders im wissenschaftlichen Bereich, mit der Bereitstellung eines Zugangssystems zu Online-Informationsangeboten eine Auswahl und Qualitätskontrolle verbunden, insbesondere bei den frei verfügbaren Quellen ${ }^{6}$.

Der Erstellung solcher Zugangssysteme widmen sich zahlreiche Projekte, die die Erstellung von Clearinghouses,

${ }^{5}$ Als (positives) „Zukunftsszenario“ etwa dargestellt von Gerhard Schneider anlässlich der 2. Inetbib-Tagung (Schneider, Gerhard: Anforderungen des wissenschaftlichen Nutzers an den Internet-Dienst der Bibliotheken. In: Weiter auf dem Weg zur virtuellen Bibliothek! Praxis, Projekte, Perspektiven. 2. Inetbib-Tagung der Universitätsbibliothek Dortmund vom 10.-11. März 1997 in Potsdam, 2. erw. Aufl., hrsg. von Beate Tröger und Hans-Christoph Hobohm. Dortmund 1997. S. 12).

${ }^{6}$ S. dazu Bargheer, Margo: Qualitätskriterien und Evaluierungswege für wissenschaftliche Internetressourcen - Ein Report für die bibliothekarische und dokumentarische Praxis <http://webdoc.sub.gwdg.de/ebook/aw/2003/ bargheer/v10.pdf>. 
Fachinformationsportalen oder Virtuellen Fachbibliotheken zum Ziel haben?.

In der Max-Planck-Gesellschaft fehlte bisher ein umfassender Wegweiser zu den vorhandenen elektronischen Informationsquellen. Vorhanden waren Insellösungen an einzelnen Max-Planck-Instituten sowie Linksammlungen zentraler Stellen zu den institutsübergreifend zugänglichen Angeboten; außerdem boten die Oberflächen der Datenbanksysteme OVID, STN und Web of Science Möglichkeiten der datenbankübergreifenden Suche und, im Falle von OVID, die Möglichkeit der Recherche nach „Local Holdings“, also in den Bibliotheken vorhandenen Beständen zu Zeitschriftentiteln. Ein allgemeines, institutsübergreifendes und benutzerfreundliches Zugangssystem zur Gesamtheit der verfügbaren Informationsquellen war nicht vorhanden.

Die Lücke sollte mit der Bereitstellung einer Virtuellen Bibliothek für die Max-Planck-Gesellschaft geschlossen werden, indem diese einen integrierten Zugang sowohl zu den gedruckten als auch den elektronischen Materialien möglichst aller zugehörigen Institutionen bietet und zudem Mehrwertdienste wie $z$. B. eine gemeinsame Suche über diese Quellen und die Verlinkung zu Volltexten und weiteren Recherchemöglichkeiten zur Verfügung stellt.

\subsection{Informationsinfrastruktur in der Max-Planck- Gesellschaft}

Die Max-Planck-Gesellschaft (MPG) betreibt Grundlagenforschung auf zahlreichen Gebieten der Wissenschaft. Ihre 80 Forschungsinstitute sind nach Fachgebieten in drei Sektionen aufgeteilt, die geisteswissenschaftliche (GW), biologisch-medizinische (BM) und die chemisch-physikalisch-technische (CPT) Sektion. Die Einrichtungen befinden sich an verteilten Standorten und repräsentieren ein breites Spektrum wissenschaftlicher Fachgebiete ${ }^{8}$.

Es gibt jedoch auch zahlreiche fachliche Überschneidungen zwischen verschiedenen Max-Planck-Instituten, zumeist innerhalb der Sektionen, aber auch sektionsübergreifend, insbesondere weil an vielen Instituten auf Grenzgebieten zwischen Forschungsrichtungen, häufig auch an interdisziplinären Themen gearbeitet wird, (das belegen etwa Institutsnamen wie „biophysikalische Chemie“, „chemische Physik fester Stoffe“ oder „Psycholinguistik“).

Eine Besonderheit in der Organisationsstruktur der MaxPlanck-Gesellschaft ist die sehr große Autonomie der einzelnen Institute, die ihre Forschung und Verwaltung traditionell in Eigenverantwortung und mit nur geringen zentralen Vorgaben organisieren. Diese weitgehende Unabhängigkeit von der zentralen Verwaltung der MPG gehört zu den wesentlichen Grundsätzen dieser wissenschaftlichen Organisation: Sie ist Teil des nach Adolf von Harnack benannten Prinzips ${ }^{9}$, nach dem die Max-PlanckInstitute anhand der Vorgaben und dem speziellen Bedarf der sie leitenden herausragenden Wissenschaftler aufgebaut und organisiert werden sollen, um so die optimalen Bedingungen für ihre jeweiligen Forschungsarbeiten zu schaffen. Aus diesem Grunde sind auch die den Instituten angeschlossenen Service- und Verwaltungseinrichtungen wie z. B. die Institutsbibliotheken, sehr unterschiedlich organisiert und ausgestattet.

So unterscheiden sich die verschiedenen Institute und Forschungsstellen in der MPG auch stark hinsichtlich der Art und Weise, wie die Versorgung der dort arbeitenden
Wissenschaftlerinnen und Wissenschaftler mit Fachliteratur und wissenschaftlicher Information organisiert ist. Um in diesem Bereich durch Kooperationen und gemeinsame Standards Verbesserungen und Synergien zu schaffen, wurden mehrere institutsübergreifende Initiativen ins Leben gerufen, die in den meisten Fällen von zentralen Einrichtungen koordiniert werden. Aktivitäten dieser Art haben in den letzten Jahren angesichts der Entwicklung des Informationsmarktes erheblich an Bedeutung gewonnen. Die zentralen und dezentralen Institutionen und Gremien, die für die Informationsversorgung in der MPG zuständig sind, werden im Folgenden kurz vorgestellt.

\subsubsection{Beteiligte Stellen und Gruppierungen}

\section{Institutsbibliotheken und Sprecherrat}

75 Max-Planck-Institute ${ }^{10}$ verfügen über eine Bibliothek. Es handelt sich dabei um wissenschaftliche Spezialbibliotheken, deren Bestand entsprechend den am jeweiligen Institut vertretenen Forschungsgebieten aufgebaut ist und deren Aufgabe die Versorgung der am Institut beschäftigten Wissenschaftlerinnen und Wissenschaftler mit der für ihre Forschung relevanten Information ist. Dabei spielen einige der Bibliotheken auch über die Max-Planck-Gesellschaft hinaus für ihr Fachgebiet eine wichtige Rolle, wie z. B. die Bibliothek des Max-Planck-Instituts für Völkerrecht in Heidelberg ${ }^{11}$.

Die Bibliotheken der Max-Planck-Institute sind sehr unterschiedlich in ihrer Größe, sowohl hinsichtlich des Personals als auch ihrer Bestände. Auch hinsichtlich ihrer technischen Ausstattung und bibliothekarischen Aktivitäten unterscheiden sich die Bibliotheken stark: Das Spektrum reicht von One Person Libraries (OPL) mit kleinem Bestand bis hin zu größeren Institutionen, besonders im geisteswissenschaftlichen Bereich, mit ca. 25 Personalstellen und bis zu 600000 Monografien und mehr als 4000 Zeitschriften.

Zwischen den Bibliotheken der Max-Planck-Gesellschaft besteht eine enge Kooperation und ein ständiger Aus-

7 Zur Terminologie der Begriffe Portal, Clearinghouse, Subject Gateway und Virtuelle Fachbibliothek s. Bargheer (Anm. 6) S. 14 f.; zur Definition des Begriffs Virtuelle Bibliothek s.a. Wiesenmüller, Heidrun: Das Konzept der „virtuellen Bibliothek" im deutschen Bibliothekswesen der 1990er Jahre. Köln. 2000. S. 18 ff. Beispiele sind etwa die Virtuellen Fachbibliotheken (s. dazu <http://www.virtuellefachbibliothek.de/>), die Fachinformationsführer (z. B. Anglistik Guide, <http:// www.anglistikguide.de/>), das Projekt DBClear (<http://www. social-science-gesis.de/Forschung/Informationstechnologie/ DBClear.htm>) oder der Meta-Gateway Renardus (<http:// www.renardus.org/>).

${ }_{8}$ Max-Planck-Gesellschaft, Zahlen und Fakten, <http://www. mpg.de/ueberDieGesellschaft/profil/zahlenUndFakten/>.

9 Harnack war Gründungspräsident der Vorgängerorganisation der MPG, der Kaiser-Wilhelm-Gesellschaft. Zum HarnackPrinzip s. vom Brocke, Bernhard und Hubert Laitko (Hrsg.): Die Kaiser-Wilhelm-/Max-Planck-Gesellschaft und ihre Institute. Berlin, New York 1996.

10 Schimmer (Anm. 3) Folie 6.

11 S. etwa deren Selbstdarstellung unter <http://www.mpil.de/ de/Bibl/info.cfm>. 
tausch von Erfahrungen und Informationen. Dazu tragen die jährlichen Treffen der Bibliothekarinnen und Bibliothekare - je einmal für die gesamte MPG und getrennt nach Sektionen - sowie die Mailingliste „Minerva-liest“ bei. Um die gemeinsamen Interessen der Bibliothekare effektiver zu vertreten, ist seit 2001 ein Sprecherrat im Einsatz, dem je zwei Vertreterinnen und Vertreter der drei Sektionen angehören; als Gast nimmt der Leiter der GVIKT (siehe unten) an den Sitzungen teil. Der Sprecherrat wird für eine Amtszeit von zwei Jahren von den Bibliothekaren gewählt.

\section{Informationsvermittlungsstellen (IVS-CPT und IVS- BM)}

Für die BM- und die CPT-Sektion gibt es je eine Informationsvermittlungsstelle ${ }^{12}$; diese befassen sich mit Datenbankrecherchen für die Wissenschaftlerinnen und Wissenschaftler, führen aber auch Benutzerschulungen zu den verschiedenen Informationsangeboten oder z. B. zur Arbeit mit Literaturverwaltungssystemen durch. In letzter Zeit sind neue Anforderungen und Aufgaben auf diese Stellen zugekommen: Zunehmend werden scientometrische Analysen und Untersuchungen nachgefragt.

Die beiden Informationsvermittlungsstellen nehmen im Übrigen an sektionsübergreifenden Projekten im Bereich der Informationsversorgung teil und stellen somit ihre Fachkenntnisse der gesamten MPG zur Verfügung.

\section{Sachgebiet wissenschaftliches Informations-, Doku- mentations- und Bibliothekswesen der Generalver- waltung (GV-IKT)}

Das der Generalverwaltung der Max-Planck-Gesellschaft in München angehörende Sachgebiet ${ }^{13}$, für das auch die Bezeichnung IKT (Informations- und Kommunikationstechnik) verwendet wird, nimmt vor allem koordinierende und unterstützende Aufgaben für die Bibliotheken und Informationsvermittlungsstellen wahr. Seine drei Mitarbeiter verwalten unter anderem mehrere Mailinglisten (z. B. die bereits erwähnte Minerva-liest), führen Verhandlungen mit Verlagen und Datenbankanbietern über zentral zu beschaffende elektronische Informationsressourcen und sind für deren Verwaltung sowie die Information der Bibliothekare zuständig. Sie nehmen außerdem an Projekten im Bereich der Informationsversorgung teil, so liegt auch die Leitung des Projekts VLib bei der GV-IKT.

\section{Gesellschaft für wissenschaftliche Datenverarbeitung Göttingen e.V. (GWDG)}

Die GWDG ${ }^{14}$ wurde 1970 als gemeinsame Einrichtung der Max-Planck-Gesellschaft und der Universität Göttingen gegründet. Sie fungiert als Hochschulrechenzentrum für Göttingen und als eines von zwei Großrechenzentren der MPG. In ihrer letzteren Eigenschaft hat sie, zusätzlich zum Betrieb von Großrechensystemen (Parallelrechnern), Netzinfrastruktur und Backup- und Archivierungssystemen, vermehrt Aufgaben im Bereich des wissenschaftlichen Informationsmanagements, unter anderem im bibliothekarischen Bereich übernommen. Sie betreibt für die MPG unter anderem einen zentralen Bibliotheksserver unter Aleph 500, einen OVID-Server und zwei Server für die
Max Planck VLib. Neben dem Rechnerbetrieb übernimmt sie dabei auch Aufgaben im Bereich der Anwendungsprogrammierung und Benutzerberatung.

\section{Beratender Ausschuss für Informationsversorgung in der MPG (BI)}

1999 wurde der BI gegründet. Dieser Ausschuss, in dem Institutsdirektoren aus allen drei Sektionen der MPG (GW-, BM- und CPT-Sektion) sowie die Vertreter des Sachgebiets wissenschaftliches Informations-, Dokumentationsund Bibliothekswesen in der Generalverwaltung der MPG (GV-IKT) vertreten sind ${ }^{15}$, wurde mit dem Ziel gegründet, die Versorgung der MPG mit elektronischer Information zu unterstützen und zu verbessern.

Eine der Aufgaben des BI besteht in der Organisation einer Grundversorgung der Max-Planck-Institute mit elektronischen Zeitschriften und Datenbanken. Diese Ressourcen werden aus einem Solidaritätsfonds finanziert, in den alle Institute einen Prozentsatz ihres Haushalts einzahlen ${ }^{16}$. Außerdem hat der BI die Errichtung des Heinz-NixdorfZentrums für Informationsmanagement in der Max-PlanckGesellschaft (ZIM) (s. unten) geplant und begleitet.

Als externer Berater nimmt seit 2000 Richard Luce, Direktor der wissenschaftlichen Bibliothek der Los Alamos National Laboratories, an den Treffen des BI teil. Er wurde zu Rate gezogen, weil seine Bibliothek, insbesondere das dort angesiedelte innovative Projekt „Library Without Walls“17, in der Versorgung der Wissenschaftler mit elektronischen Informationsressourcen internationale Maßstäbe gesetzt und damit für andere Forschungsinstitutionen und auch für die Max-Planck-Gesellschaft zum Vorbild für ihre eigenen Initiativen geworden ist.

\section{Heinz-Nixdorf-Zentrum für Informationsmanagement in der Max-Planck-Gesellschaft (ZIM)}

Neben der dauerhaften Sicherung der ausreichenden Versorgung mit elektronischer Information gilt es auch, als Forschungsgesellschaft von internationaler Bedeutung die Entwicklung neuer Formen des wissenschaftlichen Informationsaustausches aktiv mitzugestalten und diese für ihre Wissenschaftler nutzbar zu machen. Dies ist eine der Aufgaben des $\mathrm{ZIM}^{18}$, das 2001 eingerichtet wurde und in mehreren Projekten an der Modernisierung und Optimierung der Informationsinfrastruktur in der MPG arbeitet. Es wurde als unabhängige Arbeitsgruppe für zunächst fünf Jahre gegründet und wird unterstützt von der Heinz-Nixdorf-Stiftung. Als Leitbild für die Arbeit des ZIM formulierte der $\mathrm{BI}$ die „Vision einer integrierten digitalen Bibliothek für die MPG unter Beibehaltung der Grund-

\footnotetext{
12 IVS-BM <http://www.biochem.mpg.de/iv/>, und IVS-CPT $<$ http://www.mpi-stuttgart.mpg.de/ivs/IVS.html>

13 <http://www.mpg.de/ressourcenKooperationen/ informationsmanagement/generalverwaltung/>.

$14<$ <ttp://www.gwdg.de>

15 Die Mitglieder des BI sind aufgeführt unter <http://www.zim. mpg.de/Public/staff.html>

${ }_{16}$ Zur Grundversorgung s. u.

17 <http://lib-www.lanl.gov/lww/welcome.html>

$18<$ http://www.zim.mpg.de>.
} 
struktur verteilter Bibliotheken an den einzelnen MaxPlanck-Instituten“19.

Das ZIM ist nicht so sehr als operativ tätige Institution konzipiert, vielmehr liegt seine Aufgabe darin, die verschiedenen bereits existierenden Projekte im Bereich Informationsmanagement zu vernetzen und zu koordinieren sowie neue Projekte in diesem Bereich zu planen. Die praktische Umsetzung der Projektaufgaben liegt jedoch im Wesentlichen bei den anderen Einrichtungen, die in den Bereichen Informationsversorgung und -management in der MPG tätig sind, also Bibliotheken, Informationsvermittlungsstellen, GWDG und GV.

Seit seiner Gründung hat das ZIM die Aufsicht über das bereits zuvor begonnene Projekt Max Planck Virtual Library übernommen, dessen Aktivitäten es in Zusammenarbeit mit dem GV-IKT koordiniert. Ein weiteres wichtiges Projekt des ZIM ist der Dokumentenserver für Publikationen der Wissenschaftlerinnen und Wissenschaftler der MPG ${ }^{20}$. Darüber hinaus soll das ZIM für die Bekanntmachung der Neuentwicklungen in der Informationsversorgung sowohl innerhalb als auch außerhalb der Max-Planck-Gesellschaft sorgen und für diese Angebote werben. Zu diesem Zweck führt das ZIM Informationsveranstaltungen durch und konzipiert Werbematerialien; engagiert sich aber auch in nationalen und internationalen Initiativen wie $\mathrm{DINI}^{21}$ oder der Open Archives Initiative (OAI); es war an der Organisation der Open-Access-Konferenz in Berlin im Oktober 2003 beteiligt $^{22}$.

\section{Information Resource Council (IRC)}

$\mathrm{BI}$, Sprecherrat und die Informationsvermittlungsstellen sowie ein Mitglied des ZIM bilden gemeinsam das IRC. Seine Aufgabe bestand ursprünglich ausschließlich in der Beratung des ZIM; die Zusammenkünfte von Vertretern aller maßgeblichen an der Informationsinfrastruktur in der MPG beteiligten Gruppen und Instanzen sollten deren Ziele und Interessen abstimmen und dem ZIM als Unterstützung seiner Arbeit vermitteln. Mittlerweile hat sich allerdings aufgrund von aktuellen Entwicklungen der Fokus des IRC erweitert; das Gremium befasst sich nunmehr auch mit allgemeineren Fragen der Informationsversorgung in der Max-Planck-Gesellschaft, so etwa mit der Entwicklung der Grundversorgung.

\subsubsection{Informationsressourcen in der Max-Planck-Ge- sellschaft}

Den Wissenschaftlern der Max-Planck-Gesellschaft stehen verschiedene elektronische Informationsressourcen zur Verfügung. Sie werden teils von den einzelnen Instituten, teils von zentraler Stelle angeboten ${ }^{23}$. Die wichtigsten werden hier kurz beschrieben.

\section{Bibliothekskataloge}

Anhand von Bibliothekskatalogen können sich die Wissenschaftlerinnen und Wissenschaftler über die gedruckten Bücher und Zeitschriften ihrer Institutsbibliothek informieren. Dabei werden in den einzelnen Bibliotheken unterschiedliche Systeme eingesetzt. Während einige Bibliotheken ausschließlich mit gedruckten Katalogen arbeiten, verwenden die meisten EDV-gestützte Systeme. Diese variieren von selbst entwickelten Versionen, die auf Datenbanksystemen wie z. B. Access basieren, bis hin zu integrierten Lösungen wie Libero oder Aleph 500. Eine Reihe von Bibliotheken setzt im Zusammenhang mit einer Verbundteilnahme die vom jeweiligen Verbund empfohlene Software ein. Die in der MPG am weitesten verbreiteten Bibliothekssysteme sind derzeit Aleph 500 (29 Bibliothe$k^{24}{ }^{24}$ ), PICA (13), Allegro (8) und Libero (7) ${ }^{25}$.

In den EDV-Katalogen sind nicht immer die kompletten Bestände der Bibliotheken nachgewiesen; manchmal sind z. B. die Zeitschriften einer Bibliothek oder auch die Bestände bestimmter Abteilungen nicht enthalten und nur über andere Nachweisinstrumente (z. B. Kardex) zu recherchieren.

\section{Grundversorgung}

Die 1999 erfolgte Einrichtung der Grundversorgung mit elektronischen Zeitschriften und Datenbanken ist ein erster, wichtiger Schritt zu einer institutsübergreifenden Zusammenarbeit bei der Beschaffung und Bereitstellung von Informationsressourcen. Die Grundversorgung beruht auf dem Solidaritätsprinzip und wird anteilig von den Max-Planck-Instituten mit einem gewissen Prozentsatz ihres Haushalts finanziert.

Die MPG tritt bei den Verhandlungen um die Lizenzen für diese Ressourcen als Konsortium auf, womit sie ihre Position gegenüber Einzelverhandlungen der Institute stärkt und zumeist bessere Konditionen erhält. Sie erwirbt dabei Nutzungslizenzen, die für alle Einrichtungen in der MPG gelten. Die Konsortialverträge werden im Falle der e-Journals auf Basis der in den verschiedenen Instituten vorhandenen Abonnements von Printzeitschriften der jeweiligen Verlage ausgehandelt; dies erweist sich als zunehmend problematisch, da viele Institute aufgrund von Preissteigerungen und Haushaltskürzungen zu Abbestellungen gezwungen sind und dies in manchen Fällen Sanktionen seitens der Verlage für das gesamte Konsortium nach sich zieht. Die Verhandlungen mit einigen Zeitschriftenverlagen haben sich insbesondere im laufenden Jahr als sehr schwierig erwiesen, so dass das Modell der Grundversorgung in seiner gegenwärtigen Form nicht mehr als gesichert gelten kann. Mögliche Lösungen

19 Zitiert nach einem internen Papier. S. auch Velden, Theresa: Introducing ZIM / Vorstellung des ZIM. Präsentation beim „Arbeitskreis Bibliotheks- und Informationswesen der HGF“, 28. November 2001 <http://www.zim.mpg.de/presentations/ 2001Nov28Juelich/index.html>.

20 Zum Projekt eDoc s.u.

${ }^{21}$ Mitgliedschaft der Leiterin des ZIM im DINI-Beirat seit Oktober 2003.

$22<$ http://www.zim.mpg.de/openaccess-berlin/>.

${ }^{23} \mathrm{~S}$. die Übersicht unter <http://www.mpg.de/ ressourcenKooperationen/informationsservices/>.

24 Die Bibliotheken nutzen einen zentralen Aleph-Server mit Standort in der Gesellschaft für wissenschaftliche Datenverarbeitung Göttingen (GWDG); jede Bibliothek nutzt dabei ihr eigenes System mit individuellen Konfigurationseinstellungen.

25 Informationen aus einer internen Website zum VLib-Projekt. 
für diese Problematik werden derzeit in der Max-PlanckGesellschaft diskutiert.

\section{Datenbanken}

Zahlreiche Datenbanken stehen den Wissenschaftlern der MPG im Rahmen der Grundversorgung oder durch Lizenzverträge einzelner Institute zur Verfügung. Sie werden unter verschiedenen Datenbanksystemen vorgehalten; die beiden wichtigsten sind derzeit OVID, ISI Web of Knowledge und Cambridge Scientific Abstracts (CSA). Die MPG betreibt einen eigenen OVID-Server bei der GWDG $^{26}$. Die dort verfügbaren Datenbanken gehören zu den Fachgebieten Physik, Elektrotechnik, Elektronik (INSPEC), Biowissenschaften (BIOSIS), Medizin (MEDLINE, CANCERLIT), Pharmazie (EMBASE), Psychologie, Psychiatrie, Landwirtschaft, Soziologie, Erziehungswissenschaften und Wirtschaftswissenschaften. Darüber hinaus enthält OVID die fachübergreifenden Datenbanken Bowkers Books in Print, Current Contents, Dissertation Abstracts, Ulrichs International Periodicals Directory sowie MPG-spezifische Datenbanken, z. B. den Max Planck Research Report. Die beiden Chemie-Datenbanken Beilstein und Gmelin liegen unter einem bei der GWDG installierten Inhouse-System, Crossfire, auf ${ }^{27}$.

Die von Cambridge Scientific Abstracts (CSA) angebotenen Datenbanken sowie die Angebote des Web of Knowledge werden im Gegensatz dazu auf Servern der Anbieter genutzt. CSA bietet Datenbanken aus verschiedenen Fachgebieten aus dem naturwissenschaftlichen und geisteswissenschaftlichen Bereich ${ }^{28}$. Die Ressourcen des Web of Knowledge umfassen ISI Web of Science (WoS), BIOSIS Previews, ISI Proceedings sowie den ISI Journal Citation Report (JCR) ${ }^{29}$.

Weitere in der MPG genutzte Informationsquellen sind etwa INSPEC, WISO-NET oder Beck Online ${ }^{30}$.

\section{Elektronische Zeitschriften ${ }^{31}$}

Einen Zugang zu den MPG-weit lizenzierten E-Journals sowie frei zugänglichen wissenschaftlichen Zeitschriften bietet die Elektronische Zeitschriftenbibliothek (EZB); sie ermöglicht direkten Zugriff auf diese Titel unabhängig vom jeweiligen Verlag und von dem System, unter dem die jeweilige Zeitschrift vorgehalten wird. Die Daten aus der EZB liegen auch im zentralen Zeitschriftenverzeichnis der MPG vor ${ }^{32}$.

\section{Informationsangebote einzelner Institute}

Weitere Datenbanken, e-Journals und andere elektronische Informationsquellen, z. B. Reference Works, werden von einzelnen Max-Planck-Instituten lizenziert und ihren Mitarbeitern und Gästen zur Nutzung angeboten. Hinzu kommen eigene Angebote, z. B. Datenbanken über die Publikationen der am Institut arbeitenden Wissenschaftler oder eigene Publikationsprojekte wie etwa das vom Max-Planck-Institut für Gravitationsphysik publizierte e-Journal Living Reviews in Relativity ${ }^{33}$. Diese Angebote sind bisher oft nur über die jeweilige Instituts- bzw. Bibliothekshomepage, teils auch nur im Intranet des Instituts auffindbar.

\section{E-Document-Server der Max-Planck-Gesellschaft (eDoc)}

Zu den aktuellen Entwicklungen im Informationsangebot der Max-Planck-Gesellschaft gehört ein Server für elektronische Dokumente, der den Wissenschaftlerinnen und Wissenschaftlern eine Plattform für eigene Publikationen in elektronischer Form bieten möchte. Die Erschließung dieser Daten wird unter Verwendung allgemein anerkannter Metadaten-Standards durchgeführt; der eDoc-Server der MPG reiht sich damit in die internationalen Bemühungen und Initiativen zur Erstellung und Vernetzung elektronischer Archive und Publikationsserver ein $^{34}$. Der eDoc-Server ist derzeit, nach Abschluss einer Pilotphase mit sechs Instituten, mit einem Teil seiner Funktionen produktiv im Einsatz.

\section{Elektronische Bibliothek der Max-Planck-Gesellschaft (eLib)}

Aufgabe von Bibliotheken ist neben der Sammlung, Erschließung und Bereitstellung von Informationsmaterialien auch deren Archivierung mit dem Ziel, ihre dauerhafte Verfügbarkeit zu sichern. Bei elektronischen Zeitschriften, für die lediglich Nutzungslizenzen durch die Bibliotheken erworben wurden, gestaltet sich diese Aufgabe schwierig, zumal eine Langzeitarchivierung seitens der Anbieter oder Verlage in den allerwenigsten Fällen gewährleistet ist. Daher werden aktuell neue Wege der Bereitstellung und dauerhaften Sicherung von elektronischen Informationsmaterialien gesucht.

War es bisher in der Max-Planck-Gesellschaft üblich, durch Lizenzverträge den Zugang zu den Informationen auf den Servern der Anbieter zu erwerben, sollen künftig die Daten der Volltexte bezogen und auf einem eigenen Server zur Nutzung angeboten sowie archiviert werden. Vorbild für dieses Projekt ist die Library without Walls in Los Alamos. Hier werden „von den Verlagen und Datenbankanbietern nur die Rohdaten eingekauft [...], um dann unter einer einheitlichen Nutzeroberfläche aufbereitet zu werden. Dieser hochgradig integrative Ansatz erlaubt nicht nur ein Höchstmaß an Einheitlichkeit in der Präsentation der Daten, sondern auch die Entwicklung und den Einsatz auf die eigenen Bedürfnisse zugeschnittener Serviceleistungen und Werkzeuge [...]." 35

$26<$ http://ovid.gwdg.de>.

$27<$ http://www.mpi-stuttgart.mpg.de/ivs/Crossfire/beilstein. html>, <http://www.mpi-stuttgart.mpg.de/ivs/Crossfire/ gmelin.html>

$28<$ http://www.db-hosts.mpg.de/csa/csainfo.html>.

${ }_{29}<$ http://www.db-hosts.mpg.de/WoS/>.

30 Zu den verfügbaren Datenbanksystemen siehe: <http://www.mpg.de/ressourcenKooperationen/ informationsservices/datenbanken/>

31 Zu Fachzeitschriften in der MPG s. auch <http://www. mpg.de/ressourcenKooperationen/informationsservices/ zeitschriften/index.html>

32 S. Kap. 4.1.1.

$33<$ http://www.livingreviews.org/>.

$34<$ http://edoc.mpg.de/>. S. besonders die Selbstdarstellung unter <http://edoc.mpg.de/doc/help/edoc_info.epl>.

35 Zitiert nach einem nicht öffentlichen Protokoll einer Sitzung am 4.5.2000. 
Ein ähnliches Informationsangebot soll mit dem eLib-Server der Max-Planck-Gesellschaft erstellt werden, der zurzeit geplant wird.

\section{Vom „Gemeinsamen Web-OPAC“ zur Virtual Library}

Dieses Kapitel gibt Einblick in die Konzeption der Max Planck Virtual Library und zeigt auf, welche Anforderungen, Diskussionen und Entscheidungsprozesse ihr zugrunde lagen. Es wird auf die Evaluation und Auswahl der verwendeten Software eingegangen; außerdem wird ein Überblick über die verschiedenen Schritte der Inbetriebnahme des Portals bis zur offiziellen Freigabe am 21. Oktober 2002 und die darüber hinaus durchgeführten Weiterentwicklungs- und Optimierungsarbeiten bis Ende 2003 gegeben.

Eine online verfügbare Sammlung von webbasierten Informationsressourcen unterliegt zwangsläufig Veränderungen, sei es durch Änderungen von URLs oder anderen Merkmalen der verknüpften Webangebote oder auch durch deren Wegfall oder Hinzukommen. Außerdem ist auch hinsichtlich der möglichen elektronischen Mehrwertdienste ein ständiger Fortschritt zu erwarten, dessen Resultate den Nutzern auch zugänglich gemacht werden sollen. Daher befindet sich die Max Planck VLib auch nach ihrer Inbetriebnahme in einem Entwicklungsprozess. Durch den Umstieg auf eine neue Softwareversion im August 2003 konnte sie im Vergleich zum Stand bei der offiziellen Freigabe um zahlreiche Verbesserungen und neue Funktionen erweitert werden.

Die Max Planck Virtual Library wurde im Wesentlichen von den Bibliothekarinnen und Bibliothekaren der MaxPlanck-Gesellschaft konzipiert und realisiert. Obwohl das Projekt von zentraler Stelle aus koordiniert wurde, wurden die Bibliothekare in alle Entscheidungsprozesse einbezogen. So wurde eine Fragebogenaktion unter allen Bibliotheken zu Beginn des Projekts durchgeführt, um Informationen über die Ausgangssituation und Wünsche der einzelnen Bibliotheken zu erbringen. Die Projekttreffen waren immer offen für interessierte Teilnehmer, auch wenn sie nicht zur Projektgruppe gehörten; und über die wesentlichen Entscheidungen wurde auf den Bibliothekstagungen der Max-Planck-Gesellschaft abgestimmt. Alle Diskussions- und Entscheidungsprozesse wurden in Form von Protokollen ausführlich dokumentiert, um größtmögliche Transparenz zu gewährleisten.

Auf diese Weise wurden alle Institutsbibliotheken, soweit sie dies wünschten, in die Konzeption der VLib eingebunden. Damit wurde die Nähe zum Bedarf der Wissenschaftler an den Instituten gewahrt und somit dem Harnack-Prinzip ${ }^{36}$ im Rahmen des gemeinsamen Projekts nach Möglichkeit entsprochen.

\subsection{Die Anfänge}

Zwei grundlegende Überlegungen lagen der Entwicklung eines Konzepts für ein gemeinsames, webbasiertes Informationsportal zugrunde: Zum einen bestand der Wunsch nach einem gemeinsamen Zeitschriftennachweis, um den Leihverkehr innerhalb der MPG zu erleichtern. Dieser funktionierte bereits seit längerem informell, zumeist durch Anfragen an die Mailingliste der Bibliothekare, „Minerva- liest". Eine zentrale Datenbank, die die Bestandsdaten möglichst aller in Max-Planck-Instituten gehaltenen Zeitschriften enthielte, würde den Bibliothekaren, aber auch den Wissenschaftlern die rasche Lokalisierung einer gewünschten Ausgabe ermöglichen. Gedacht war beim gemeinsamen Zeitschriftennachweis zunächst vorwiegend an die gedruckten Zeitschriften.

Zwei Institutsbibliotheken der Biologisch-Medizinischen Sektion der Max-Planck-Gesellschaft hatten überdies bereits eine gemeinsame Datenbank über ihre gesamten Bestände (Monografien und Periodika) eingerichtet, in die sie die Daten aus ihrem jeweiligen Bibliothekssystem per FTP einspielten ${ }^{37}$. Da auch die anderen Sektionen Interesse an einem gemeinsamen Bibliothekskatalog über die Monografiebestände bekundeten, wurde die Idee des gemeinsamen Katalogs erweitert; Ziel war nunmehr ein gemeinsames Nachweisinstrument über den (gedruckten) Zeitschriften- und Monografienbestand (möglichst) aller Bibliotheken innerhalb der MPG.

\subsubsection{Internes Nachweisinstrument oder öffentlicher Katalog}

Zunächst musste die Frage geklärt werden, ob dieser gemeinsame Katalog nur den Bibliothekaren zur Verfügung stehen würde, oder auch den Wissenschaftlern in der MPG oder, darüber hinaus, der allgemeinen Öffentlichkeit zugänglich sein sollte. Ein Interesse der Wissenschaftler an einem solchen Katalog wurde anfangs bezweifelt, da diese zumeist nur die Bestände ihrer eigenen Bibliotheken oder höchstens noch der Bibliotheken fachlich verwandter Max-Planck-Institute, nicht aber die der Gesamtheit der Bibliotheken durchsuchen würden. Möglicherweise stand hinter dieser Argumentation aber auch die Sorge, die Wissenschaftler könnten nunmehr ohne Rückgriff auf die Unterstützung durch die Bibliothek recherchieren und dabei möglicherweise die im Institut selbst vorhandenen Bestände übersehen, was mitunter zu überflüssigen Anfragen an die anderen Institute führen könnte - ähnliche Befürchtungen bestehen an einigen Bibliotheken auch hinsichtlich einer direkten Nutzung von Dokumentlieferdiensten durch die Benutzer.

Viel sprach jedoch auch für den öffentlichen Zugang zum "gemeinsamen WebOPAC" - so der Arbeitstitel der Initiative. Nach der Einrichtung der Grundversorgung zeigte sich hier ein weiterer konkreter Ansatz zur Modernisierung der Informationsversorgung und zur Kooperation der daran beteiligten Stellen in der Max-Planck-Gesellschaft. Es wurde als wünschenswert angesehen, dies auch nach außen hin zu dokumentieren. Daher wurde der WebOPAC auch als informationspolitisches Signal gewertet und seine öffentliche Sichtbarkeit vom BI empfohlen.

Es wurde daher beschlossen, dass der Katalog generell öffentlich sichtbar und zugänglich sein sollte, dass aber auf Wunsch einzelner Bibliotheken ihre Bestände darin nur Angehörigen der Max-Planck-Gesellschaft angezeigt werden sollten. Für das Projekt hatte diese Entscheidung

36 S. Kap. 2.2.

37 Erreichbar über den Bibliothekskatalog des Fritz-Haber-Instituts <http://root.rz-berlin.mpg.de/bib/>. 
die Konsequenz, dass bei der Auswahl einer geeigneten Software die Möglichkeit berücksichtigt werden musste, für Teile des Gesamtbestandes die Recherche und Anzeige auf bestimmte, berechtigte Benutzerkreise zu beschränken - es musste ein System gefunden werden, das die Möglichkeit enthielt, verschiedene Sichten auf den Bestand zu generieren.

\subsubsection{Das „hybride Modell“}

Eine weitere grundlegende Frage war, ob die Zeitschriften- und Monografienbestände in einen gemeinsamen, zentral gehaltenen Datenbestand geladen werden sollten. Dieser würde durch Upload der Daten aus den verschiedenen lokal eingesetzten Bibliothekssystemen aktualisiert werden. Als Alternative bot sich eine dezentrale Lösung mit einem „virtuellen Katalog“ an, wie sie - in unterschiedlicher Weise - etwa beim Karlsruher Virtuellen Katalog (KVK) oder beim Kooperativen Bibliotheksverbund Berlin-Brandenburg (KOBV) realisiert war.

In beiden Fällen würde sich die Aufgabe stellen, verschiedene Formate aufeinander abzubilden - im ersten Fall beim Laden der Daten aus den verschiedenen Ausgangssystemen in die zentrale Datenbank, im zweiten Fall bei der Konfiguration der Suchmaschine für einen Metakatalog.

Dabei schien die erste Möglichkeit anfangs die bessere zu sein. Insbesondere wurde argumentiert, dass die Qualität des Katalogs und der darin angebotenen Services bei der Überführung der Bestände in eine zentrale Datenbank nicht von der Qualität und den Möglichkeiten der lokalen Kataloge abhängig wäre und dass außerdem die Zugriffe auf den Gesamtkatalog die lokalen Systeme nicht belasten würden.

Als nachteilig an dieser Lösung wurde jedoch der zu erwartende große Arbeitsaufwand bei der Konfiguration der Schnittstellen, der regelmäßigen Pflege des zentralen Systems und - für die Bibliotheken wie auch den Datenbankbetreiber - beim regelmäßigen Upload der Daten gewertet.

Favorisiert wurde schließlich eine Lösung, für die sich der Sprachgebrauch „hybrides Modell“ einbürgerte. Sie bestand darin, die Zeitschriften, wie ursprünglich geplant, in einen zentralen Katalog zu importieren, für die Monografien jedoch einen Suchmechanismus über die verschiedenen, dezentral gehaltenen Kataloge einzurichten.

Für die Zeitschriftenbestände wurde eine zentrale Datenhaltung befürwortet, um eine hohe Qualität der Daten zu erzielen und die Einrichtung von elektronischen Mehrwertdiensten zu ermöglichen. Die in den Ausgangssystemen vorhandenen Katalogisate waren von sehr unterschiedlicher Qualität und enthielten z. B. nicht immer eine ISSN, was eine eindeutige Zuordnung eines Zeitschriftentitels erschwerte. Die Zusammenführung der Daten in einen gemeinsamen Katalog gestaltete sich aus diesem Grund zunächst schwierig. Ein probeweiser Import der Daten in eine Oracle-Datenbank hatte ergeben, dass eine große Anzahl an Dubletten entstand, die nur mit unverhältnismäßig großem Arbeitsaufwand und - wegen der zum Teil fehlenden ISSN - zweifelhaften Ergebnissen hätten bereinigt werden können.

Eine Lösung für dieses Problem bot sich durch die Zusammenarbeit mit der Zeitschriftendatenbank (ZDB). Ein Teil der Institutsbibliotheken meldeten bereits ihre Zeit- schriftenbestände dorthin, entweder durch Zugehörigkeit zu einem Verbund oder in Eigenregie. Der Vorteil einer ZDB-Teilnahme liegt in der Nutzung der dort enthaltenen, autoritativen Daten, die im Rahmen von Lieferdiensten den teilnehmenden Bibliotheken für die eigene Nutzung zur Verfügung gestellt werden können. Eine Teilnahme möglichst aller Bibliotheken in der Max-Planck-Gesellschaft an der ZDB bot somit die besten Voraussetzungen, mit relativ geringem Aufwand zu guten Resultaten zu gelangen. Verhandlungen mit der ZDB ergaben, dass diejenigen Zeitschriftentitelsätze, mit denen Bestände von Max-Planck-Instituten verknüpft sind, in einem halbjährlichen Rhythmus im MAB2-Format geliefert werden konnten. Diese Daten konnten dann in ein Standard-Bibliothekssystem importiert und als MPG-Zeitschriftenkatalog bereitgestellt werden. Zudem wurde auch eine Lieferung der für die Max-Planck-Gesellschaft relevanten Bestände aus der EZB angeboten, so dass der Zeitschriftenkatalog nicht nur die gedruckten, sondern auch die elektronischen Bestände enthielte.

Die Teilnahme der Bibliotheken an der ZDB wurde daher - auf freiwilliger Basis - nach und nach ausgebaut; mittlerweile melden ca. 35 Institutsbibliotheken ihre Bestände an die ZDB.

\subsubsection{Ausbau zur Virtuellen Bibliothek}

Zu diesem Zeitpunkt wurde auch bereits daran gedacht, ein System einzurichten, das ausbaufähig in Richtung auf ein in einem Positionspapier als „Digitale Bibliothek der MPG" bezeichnetes Informationsangebot sein sollte, das nicht nur die Bibliothekskataloge und das geplante Zeitschriftenverzeichnis umfassen sollte, sondern ein möglichst vollständiges einheitliches Zugangssystem zu den in der Max-Planck-Gesellschaft vorhandenen Informationsressourcen bieten sollte.

Zwar hatte 1999 eine Arbeitsgruppe den Bedarf an einem zentralen Zugangssystem für die Max-Planck-Gesellschaft diskutiert und war zu dem Schluss gekommen, es sei (noch) nicht erforderlich, ein solches System einzurichten. Davon wurde im Gegenteil sogar abgeraten, weil die Qualität der zu dieser Zeit verfügbaren Suchoberflächen eine deutliche Verschlechterung gegenüber den bereits in der MPG vorhandenen mit sich brächte ${ }^{38}$. Für die Einführung sprach jedoch der von den Bibliothekaren festgestellte Bedarf. Überdies waren inzwischen mit der KOBV-Suchmaschine und der Digitalen Bibliothek Nordrhein-Westfalen qualitätsvolle Systeme an den Start gegangen, die auf eine erfolgreiche Realisierung eines eigenen Systems schließen ließen.

Im Verlauf des Projekts wurde statt der Arbeitsbezeichnung Digitale Bibliothek der Name Virtuelle Bibliothek der Max-Planck-Gesellschaft / Max Planck Virtual Libra-

38 Bowman, Benjamin F.: Stellungnahme für das Projekt Informationsversorgung. Thema: „Zugangssystem“ <http://w3. rz-berlin.mpg.de/infoprojekt/zugang.html>. 
ry gewählt ${ }^{39}$. Mit diesen Vorgaben wurde mit der Vorbereitung der technischen Umsetzung begonnen, insbesondere mit der Evaluation angebotener Software- und Servicelösungen.

\subsection{Produktauswahl für die Max Planck Virtual Li- brary}

Ein vorläufiger Anforderungskatalog an ein solches System enthielt unter anderem:

- Auswahlmöglichkeit der angebotenen Bibliothekskataloge (nach Fachgebieten, Sektionen bzw. individuell zusammengestellten Katalogen),

- Anzeige der Ergebnissätze in einheitlicher Form und mit Dublettenkontrolle,

- direkte Verlinkung vom Rechercheergebnis zur entsprechenden Fundstelle im jeweiligen Katalog.

Wie bereits erwähnt, sollte die Ausbaufähigkeit zu einem Informationssystem im Sinne einer Virtuellen Bibliothek für die MPG bzw. Integrationsmöglichkeit in ein solches System gegeben sein.

\subsubsection{Standardprodukt oder Eigenentwicklung}

Als Möglichkeiten der Realisierung standen grundlegend zwei Möglichkeiten zur Wahl: Um ein maßgeschneidertes System zu erhalten, das den Ansprüchen der MaxPlanck-Gesellschaft besonders gut gerecht werden würde, konnte eine Eigenentwicklung vorgenommen werden. Um personelle Ressourcen, speziell für die Programmierung und Weiterentwicklung eines solchen Systems, zu sparen und kontinuierlichen Support und Wartung der Software zu gewährleisten, konnte auf ein bereits am Markt vorhandenes Produkt zurückgegriffen werden.

Mit einer selbst entwickelten Lösung konnte neben der genauen Abstimmung auf die Anforderungen der MPG auch, besonders durch den Einsatz von Open-SourceKomponenten, eine Unabhängigkeit von kommerziellen Anbietern erzielt werden ${ }^{40}$.

Vorteil eines Standardproduktes wäre demgegenüber, dass ein fertiges und bereits erprobtes Produkt eingesetzt würde. Für die Programmierung wären keine eigenen Arbeiten auf Kundenseite erforderlich; in der Regel muss ein fertiges System lediglich konfiguriert werden, wobei auch dies durchaus aufwändig sein kann. Es kann neben dem Produkt und den Nutzungslizenzen auch der notwendige Support, Wartung und Weiterentwicklung mit eingekauft werden. Besonders bei einer am Markt bereits etablierten Firma kann von einer dauerhaften Unterstützung ausgegangen werden. Nicht zu vernachlässigen sind auch Synergieeffekte durch Kontakte zu anderen Kunden, z. B. in Form von Mailinglisten und User Groups und den Austausch von Erfahrungen, Arbeitshilfen sowie eventuell zusätzlichen, selbst entwickelten Programmkomponenten, ein gemeinsames Auftreten gegenüber der Firma kann bei der Realisierung eigener Wünsche hilfreich sein. Bei einem bereits bekannten System kann möglicherweise auch durch die Vertrautheit der Benutzer mit anderen mit dem gleichen Produkt realisierten Angeboten eine leichtere Nutzbarkeit durch Wiedererkennungseffekte gegeben sein.

In der MPG fiel die Entscheidung zugunsten eines Standardproduktes aus, nicht zuletzt aufgrund der relativ ge- ringen personellen Ausstattung in den beteiligten Institutionen (Bibliotheken, ZIM, GV, GWDG). Der Vorteil einer kompetenten und am Markt etablierten Firma als Partner für Support, Wartung und Weiterentwicklung war einer der wichtigsten Faktoren für diese Entscheidung.

Im Übrigen war die Entwicklung des Marktes zum Zeitpunkt der Planung der Max Planck VLib soweit vorangeschritten, dass es bereits einige vergleichbare wissenschaftliche Informationsportale (DigiBib NRW, Library without Walls; mit Einschränkungen vergleichbar auch KOBV, KVK) gab, an denen man sich orientieren und von deren Erfahrungen, die in die Entwicklung der zur Verfügung stehenden Softwareprodukte eingeflossen waren, profitiert werden konnte.

Sowohl im Falle einer Eigenentwicklung als auch bei Verwendung eines Standardprodukts würden bestimmte Anforderungen an die Lokalsysteme gestellt. Diese müssten mindestens über eine WWW-Schnittstelle verfügen, besser noch das Z39.50-Protokoll unterstützen. Aus Sicht der Lokalsysteme waren somit die Anforderungen in beiden Fällen in etwa gleich.

\subsubsection{Begutachtete Produkte}

Nach ersten Überlegungen wurden vier verschiedene Softwarelösungen, die in zwei Fällen als Gesamtlösung mit Hosting angeboten wurden, in Augenschein genommen: der Karlsruher Virtuelle Katalog (KVK), der Gemeinsame Bibliotheksverbund (GBV) mit dem PICA-System, die Produkte Metalib und SFX der Firma Ex Libris sowie die Information Portal Suite (IPS) der Firma IHS ${ }^{41}$.

Während die ersteren beiden Angebote Komplettlösungen mit Hosting auf Servern der Anbieter und relativ umfassender Betreuung darstellten, umfassten die beiden kommerziellen Angebote nur die Lieferung der Software mit den dazugehörigen Lizenzen sowie Unterstützung bei der Implementierung und Konfiguration des Produkts. Durch Wartungsverträge würde der kontinuierliche Support und der Bezug von Systemaktualisierungen geregelt.

39 Die Abgrenzung der beiden Begriffe Digitale und Virtuelle Bibliothek wird nicht eindeutig vorgenommen. R. Hacker betrachtet sie als „im Kern“ gleichbedeutend; die Unterschiede seien eher graduell (Hacker, Rupert: Bibliothekarisches Grundwissen. München ${ }^{7} 2000$. S. 132); ähnlich äußert sich Wiesenmüller (Anm. 7), die der „Virtuellen Bibliothek“ für ihre Untersuchung den Vorzug gibt und drei Grundmerkmale für diese formuliert: „Erstens das Vorliegen digitaler Daten in großem Stil, zweitens die durch Vernetzung erreichte Ortsunabhängigkeit, drittens der verbesserte Zugang über eine einheitliche Benutzeroberfläche sowie Mehrwertleistungen für die Benutzer" (S. 18). Diese Gegebenheiten treffen auf die Virtuelle Bibliothek der Max-Planck-Gesellschaft zu.

40 Dieser Weg wurde für den eDoc-Server der MPG gewählt.

$41 \mathrm{Vgl}$. auch die Evaluation von Portalsystemen durch den KOBV, dargestellt von Litsche, Stefan und Dagmar Otto: Evaluation ausgewählter Portalsysteme, ZIB-Report ZR 02-21. - März 2002, <ftp://ftp.zib.de/pub/zib-publications/ reports/ZR-02-21.pdf>. 
Die Angebote wurden der Projektgruppe und interessierten Zuschauern vorgestellt und anschließend eingehend diskutiert. Die Anbieter erhielten vorab einen Fragenkatalog, auf den sie bei ihren Produktvorstellungen eingingen. Die vier Angebote wiesen in ihren Grundzügen folgende Inhalte auf, wobei hier der Stand zur Zeit der Präsentationen wiedergegeben ist, ohne später erfolgte Weiterentwicklungen zu berücksichtigen:

\section{Gemeinsamer Bibliotheksverbund (GBV) ${ }^{42}$}

Eine Reihe von Max-Planck-Instituten nimmt bereits am Gemeinsamen Bibliotheksverbund teil. Ihre Bibliothekskataloge sind bereits im Rahmen des PICA-Gesamtkatalogs gemeinsam durchsuchbar, ähnlich wie die Regionalkataloge innerhalb des GBV.

Der GBV bot an, auch die übrigen Institute, die nicht Verbundteilnehmer sind, in diesen gemeinsamen OPAC aufzunehmen. Realisiert würde der gemeinsame Katalog mit einer zentralen Datenbank, in die ein regelmäßiger Upload der Daten der verschiedenen Bibliotheken im MAB2Format erfolgen würde. Voraussetzung wäre also eine MAB2-Exportmöglichkeit aus dem Bibliothekssystem der teilnehmenden Bibliotheken. Eine Dublettenkontrolle wäre beim Laden der Daten möglich. Der gemeinsame Opac könnte dann mit einem WWW-Browser oder mit einem Z39.50-Client durchsucht werden.

Besonders hervorgehoben wurde die Anbindung an - allerdings kostenpflichtige - Services des Verbundes wie die GBV-Lieferdienste (GBV-direkt und Online-Fernleihe) sowie die Nutzung der Datenbank Online Contents (einer Zeitschriftendatenbank, aus der direkt bestellt werden kann).

Auch die Aufnahme der ZDB-Daten könnte über den GBV erfolgen - das würde das Aufsetzen einer eigenen Datenbank ersparen. Schwierig wäre die Einbindung der elektronischen Lizenzen der MPG und die Einrichtung des Zugriffs auf die entsprechenden Zeitschriften, man würde sich jedoch um eine Lösung bemühen.

\section{Karlsruher Virtueller Katalog (KVK) ${ }^{43}$}

Der KVK funktioniert nach dem Prinzip der parallelen Suche in mehreren, vorher durch das Aktivieren von Checkboxen ausgewählten Katalogen. Aus den Einzelsystemen werden nur Kurztiteldaten geholt, um die Belastung der Einzelsysteme gering zu halten; zur Vollanzeige wird dann direkt in die lokalen Kataloge verlinkt.

Die Anzeige der Ergebnissätze erfolgt in der Reihenfolge der Antwort der abgefragten Server ohne Zusammenführung; eine Dublettenkontrolle ist daher nicht möglich. Die Sortierung der Treffer erfolgt nach den jeweiligen lokalen Einstellungen.

Technisch ist die verteilte Suche mit Hilfe eines für jeden einzelnen Katalog konfigurierten Filters realisiert, der die Suchanfrage in die jeweilige datenbankspezifische Syntax übersetzt. Die Anbindung ist in der Regel über das http-Protokoll realisiert, möglich wäre auch eine Z39.50Anbindung.

Eine Auflistung der Kataloge nach Sektionen oder anderen Kriterien ist möglich. Eine Lösung für das Zeitschriftenverzeichnis oder weitere Services waren im Rahmen des KVK-Angebotes nicht enthalten.

\section{Metalib $^{44}$}

Das Portalsystem Metalib, ein kommerzielles Standardprodukt, wurde aufgrund der Erfahrungen bei der Entwicklung des Verbundkataloges für den KOBV entwickelt, dessen Verbundkatalog nicht auf einer zentralen Datenbank, sondern auf einer gleichzeitigen Suche in den lokalen Katalogen beruht. Zum Zeitpunkt der Evaluation für die Virtuelle Bibliothek der Max-Planck-Gesellschaft waren noch keine konkreten Anwendungen zu besichtigen. Metalib wird von der Firma Ex Libris entwickelt, die auch Hersteller des Bibliothekssystems Aleph 500 ist.

Metalib führt mit einer parallelen verteilten Suche verschiedene Datenpools (nicht nur Bibliothekskataloge) unter einer Suchoberfläche zusammen. Die Suchergebnisse werden getrennt, aber unter einer einheitlichen Oberfläche in einem Standardformat angezeigt; sie können nachträglich durch eine Merge-Funktion zusammengeführt werden, wobei auch, zumindest bei kleineren Treffermengen, Dubletten bereinigt werden.

Die verteilte Suche wird mit dem Modul „Universal Gateway“ realisiert, der eine Suchanfrage über heterogene Datenquellen mit verschiedenen Zugangsprotokollen und verschiedenen Datenformaten ermöglicht. Erforderlich ist dazu für jede angeschlossene Datenbank eine Konfigurationsdatei, die Adressen, Protokolle und Mappings enthält. Die Anbindung der Datenquellen erfolgt über die Protokolle Aleph (proprietäres Protokoll), Z39.50 oder http.

Zur Verwaltung der in Metalib eingebundenen Datenbanken dient die Komponente Resource Store; dieser wird über ein Web-Interface konfiguriert, so dass an dieser Stelle keine Programmierung erforderlich ist. Bei der Katalogisierung der Ressourcen wird differenziert zwischen solchen Ressourcen, die für die Suche unter Metalib konfiguriert werden können („Search“-Ressourcen), und solchen, für die das nicht möglich ist. Letztere werden in der Oberfläche von Metalib getrennt von den Search Resources angezeigt und können durch einen Link erreicht werden, so dass unter ihrer eigenen Oberfläche recherchiert werden kann („Link to“-Ressourcen).

Die Informationen über die erfassten Ressourcen werden in einer Datenbank („Knowledge Base“) gesammelt. Diese wird mit der Metalib-Software an die Kunden ausgeliefert und regelmäßig aktualisiert. Prinzip dabei ist die Kooperation zwischen den Beteiligten: Die Institutionen können für die Realisierung ihres Portals auf bereits vorliegende Konfigurationen zurückgreifen, sofern die entsprechenden Lizenzen erworben wurden. Neue Ressourcen werden von den Institutionen konfiguriert; die fertige Konfiguration fließt zurück in die Knowledge Base und wird so an die anderen Kunden verteilt, die sie nutzen können, sofern sie dazu berechtigt sind. Nicht genutzte Ressourcen werden in der jeweiligen Metalib-Installation nicht aktiviert und sind somit für die Benutzer nicht sichtbar.

\footnotetext{
$42<$ http://www.gbv.de>.

$43<$ <ttp://www.ubka.uni-karlsruhe.de/kvk.html>.

$44<$ http://www.metalib.com/>.
} 
Ein Modul zur Nutzerverwaltung regelt die Authentifizierungen, Zugriffsrechte und Standardeinstellungen für die eingetragenen Benutzer und Gastzugänge. Eine Berechtigungssteuerung erfolgt bei allen Schritten. Möglich ist aber auch der Anschluss von Metalib an eine externe Nutzerverwaltung. Es werden für die Benutzer Dienste wie EShelf, Benachrichtigungsdienste und das Speichern der Suchhistorie angeboten.

Möglich ist die Definition von Gruppen mit jeweils zugeordneten Sichten und Rechten, so dass solche Datenquellen, auf die nicht alle Benutzer Zugriff haben, differenziert angeboten werden können.

Zusätzlich zum verteilten Katalog wird für weitere Services und Verfügbarkeitsrecherchen die Software SFX angeboten, die in die Metalib-Oberfläche, aber auch in andere Suchoberflächen - soweit diese dafür konfigurierbar sind - eingebunden werden kann. Dabei werden, ausgehend von einer Analyse des Rechercheergebnisses, kontextsensitive Links zu weiteren relevanten Informationsangeboten, und zwar über den in Metalib definierten Suchraum hinaus hergestellt. Diese Funktion würde den Mehrwert einer gemeinsamen Portallösung besonders steigern.

Metalib würde durch die Einbindung von verschiedenen, nicht nur bibliothekarischen Datenquellen über die Dimension eines gemeinsamen Web-OPACs hinausreichen und die mittelfristig projektierte Virtuelle Bibliothek ermöglichen.

\section{Information Portal Suite (IPS) ${ }^{45}$}

Auch die Information Portal Suite der Firma IHS geht über die Dimension der Angebote von KVK und GBV hinaus und ist auf einer Ebene mit Metalib zu sehen. Mit diesem Produkt ist die Digitale Bibliothek Nordrhein-Westfalen realisiert; der Gateway Bayern arbeitet mit einer Varian$t^{46}$. IPS regelt den Zugriff auf verteilte Datenquellen und ermöglicht Personalisierung und Kommerzialisierung. Im Interesse der Datensicherheit werden die übertragenen Daten mit SSL verschlüsselt.

Das Produkt besteht aus drei Modulen: IPS Community regelt die Zugangskontrolle, bei der Nutzergruppen und unterschiedliche Sichten definiert werden können. IPS Access organisiert die parallele Suche in den Zielsystemen. Für jede Datenquelle muss auch hier ein „Gateway“ konfiguriert werden Dies wird mittels Perl eingerichtet. Obwohl es sich bei IPS um eine Standardsoftware handelt, bei der laut Ankündigung nur Konfigurations-, nicht aber Programmierarbeiten auf seiten des Kunden anfallen würden, würde hier dennoch ein gewisser Programmieraufwand erforderlich. Das dritte Modul, IPS Commerce, ermöglicht ein Monitoring aller Benutzeraktivitäten, z. B. für Abrechnungen oder statistische Auswertungen. Die verteilte Suche wird mit einer Standardsoftware, Query Server von Dataware Technologies, durchgeführt; für die Anbindung werden die Protokolle http und Z39.50 unterstützt.

Auch bei IPS werden nicht nur Ergebnislisten angeboten, sondern die Daten können auch im Vollformat auf der einheitlichen IPS-Oberfläche betrachtet werden. Eine Dublettenkontrolle ist allerdings nicht möglich. Die Ergebnisse bzw. Trefferlisten können per E-Mail in einem kommagetrennten Format verschickt werden; ein direkter Import in Literaturverwaltungssysteme wird nicht angeboten.

Eine Selektion bzw. Erzeugung von Sichten nach Institu- ten, Sektionen, Regionen usw. ist möglich. Zugriffsrechte können nach den Parametern Standort, Nutzer, Sicht zugewiesen werden.

Im Gegensatz zu Metalib bot IPS, allerdings nur bei Erwerb des zusätzlichen Produkts IPS Tunnel, bereits zu diesem Zeitpunkt die Möglichkeit des IP-Tunneling, also der Weitergabe einer authentifizierten IP-Nummer an ein Endsystem (z. B. bei Nutzung von IPS auf Reisen).

Eine Verfügbarkeitsrecherche, in etwa vergleichbar mit dem SFX-Dienst bei Metalib, wird ebenfalls geboten, bietet aber offenbar nicht den gleichen Leistungsumfang.

Zusammengefasst stellten sich die Unterschiede im Leistungsumfang der einzelnen Angebote folgendermaßen dar: Der KVK bietet eine Metasuche über Bibliothekskataloge, das Angebot umfasst also genau die Realisierung einer der beiden Kernanforderungen, nämlich einen gemeinsamen „virtuellen“ Katalog über die Monografie-Bestände der Max-Planck-Gesellschaft, in den ein selbst verwaltetes Zeitschriftenverzeichnis integriert werden könnte. Der GBV bietet in etwa dasselbe und darüber hinaus die Anbindung an GBV-Umgebung und -Lieferdienste, an Online Contents usw. Dagegen sind Metalib und IPS komplexere Systeme, die über den virtuellen Katalog der Bibliotheksbestände hinaus auch die Einbindung anderer Datenquellen bieten und somit die Möglichkeit eines Ausbaus zu größeren Informationssystemen enthalten.

Bezüglich der Gestaltung der Oberflächen war bei allen vier Angeboten die Umsetzung eigener Design-Wünsche möglich; lediglich bei Metalib wurde darauf hingewiesen, dass bestimmte Grundeinstellungen der Standardoberfläche nicht veränderbar sind. Mit Ausnahme von Metalib (hier nur englische Version) wurden alle Produkte in einer zweisprachigen Oberfläche (deutsch/englisch) angeboten.

Im Laufe der Evaluationsphase kamen zusätzliche Wünsche an das gesuchte Produkt auf. So zeigte sich, dass es sinnvoll wäre, möglichst ein modular aufgebautes System zu verwenden, das eine inkrementelle Inbetriebnahme der verschiedenen Komponenten gestattet. Dies schien bei Metalib stärker der Fall zu sein als bei IPS, insbesondere eine Nutzung der SFX-Komponente ist unabhängig von Metalib möglich. Die Firma IHS bestätigte, dass IPS eher monolithisch und seine Komponenten stark ineinander verschränkt seien.

Es wurde schließlich eine Entscheidung für die Metalib-Software getroffen. Nun konnten die weiteren Schritte folgen - Vertragsverhandlungen, Anforderungen und Verantwortlichkeiten festlegen, genauere Planung des Projekts.

$45<$ http://www.i-portalsuite.de/>.

${ }^{46}$ Gillitzer, Berthold: Gateway Bayern: ein Internetportal $<$ http://gateway-bayern.bib-bvb.de/docs/akt/digibib_info. htm>, o.S. 


\subsection{Implementierungsphase und Routinebetrieb}

\subsubsection{Aufgaben im Projekt}

Das Konzept der Max Planck VLib wurde bei Informationsveranstaltungen MPG-intern, aber auch öffentlich vorgestellt (s. Abb. 1).

Die Inbetriebnahme von Metalib und SFX erforderte eine Reihe von Arbeitsschritten, die nun kurz skizziert werden sollen. Diese Aufgaben werden von einer aus derzeit acht Personen bestehenden Arbeitsgruppe erledigt, die jeweils mit einem Teil ihrer Arbeitszeit für das Projekt eingesetzt wurden. Dieses Projektteam wird von einer Beratungsgruppe mit elf Mitgliedern unterstützt.

\section{Hardware und Systemvoraussetzungen}

Die Hardware für die VLib, zunächst ein Server unter Solaris, wurde von der GWDG beschafft und in Betrieb genommen. Mittlerweile ist diese Plattform durch Linux abgelöst worden. Zunächst wurden Metalib und SFX auf einer Maschine betrieben, während der Einführungsphase stellte sich jedoch heraus, dass aus zahlreichen Instituten nicht auf den SFX-Dienst zugegriffen werden konnte, weil der entsprechende Port am Server in den Firewalls der Institute gesperrt war. Um SFX nutzen zu können, hätte in jedem der betroffenen Institute eine Freischaltung dieses Ports erwirkt werden müssen. Um dies zu umgehen, wurden Metalib und SFX auf zwei verschiedenen Rechnern installiert, so dass nun beide über den Standard-http-Port 80 erreicht werden können und keine weiteren Bemühungen seitens der Benutzer erforderlich sind. Für die Implementierung weiterer Funktionen und die Übernahme von Software-Aktualisierungen wurden zudem für SFX und Metalib jeweils Testumgebungen geschaffen, um den Produktionsbetrieb von den Entwicklungsarbeiten zu trennen und somit Betriebsunterbrechungen zu vermeiden.

\section{Konfiguration und Katalogisierung der Ressourcen in Metalib}

Die Informationsressourcen für die Metalib-Suche wurden nach vorher festgelegten Regeln mit Hilfe der webbasierten Verwaltungsschnittstelle katalogisiert und für die Suche konfiguriert. Die Konfiguration einer Datenbank oder anderen Informationsquelle für die Suche in Metalib ist nicht nur von Metalib selbst, sondern auch von den Gegebenheiten in den Zielsystemen abhängig. Sie wurde

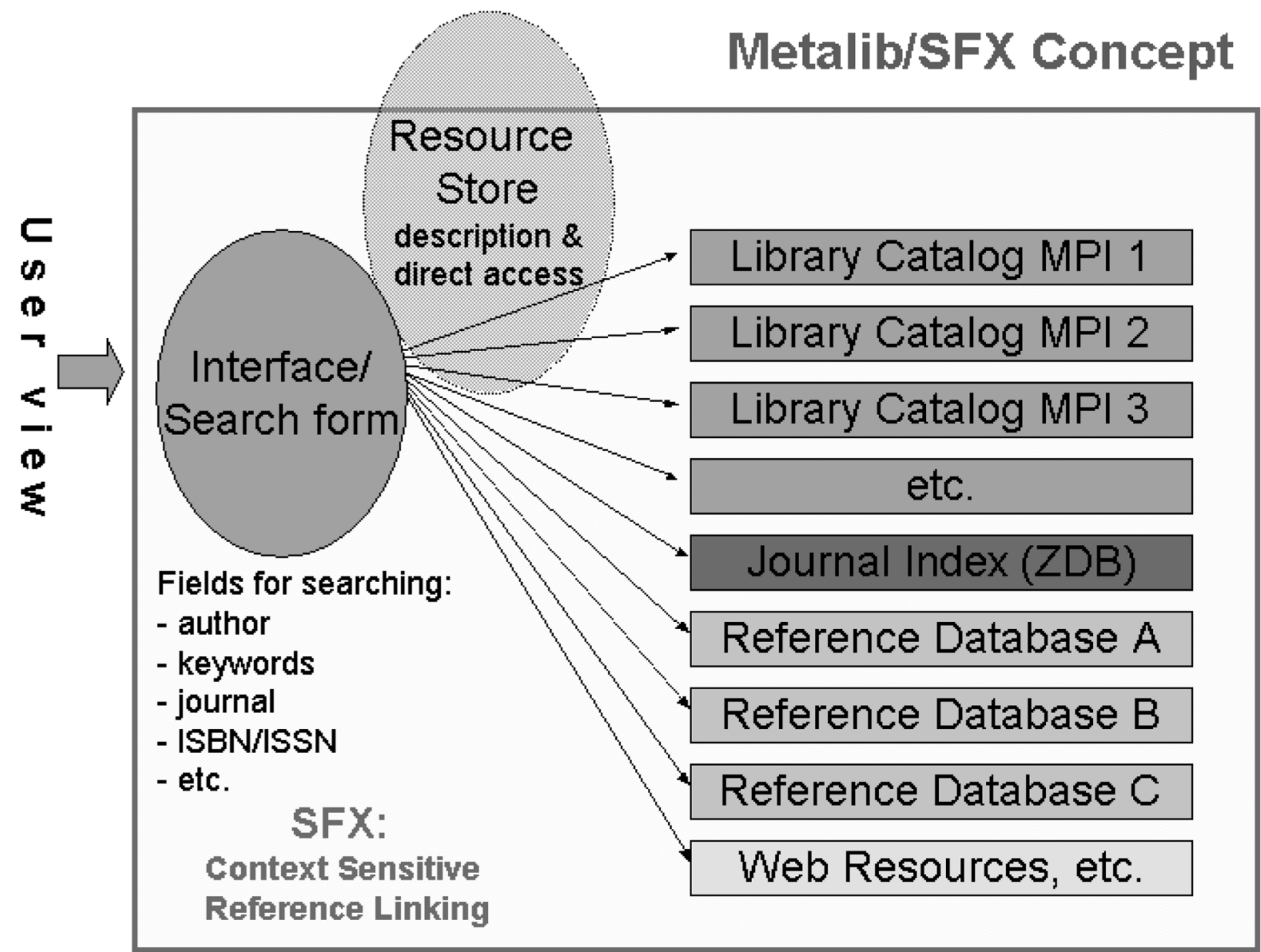

Abb. 1: Konzept der Max Planck Virtual Library. Quelle: Schimmer, Ralf: Auf dem Weg zu einem bruchlosen Informationsraum (s. Anm. 3), Folie 11. 
zunächst für die Bibliothekskataloge der Max-Planck-Institute durchgeführt und anschließend nach und nach für die übrigen Informationsquellen. Die Konfiguration der Ressourcen erforderte in einigen Fällen, wie bei ISI (z. B. Web of Science), Verhandlungen mit den Datenbankanbietern oder Verlagen, weil die Einbindung ihrer Ressourcen in das System auch von deren Kooperation abhing. In vielen Fällen erwiesen sich schließlich die Feinarbeiten, die notwendig waren, um die Suchfunktion und Ergebnisanzeigen der einzelnen Ressourcen korrekt einzurichten, als recht aufwändig.

\section{Konfiguration von SFX}

Die Einrichtung und Anpassung von SFX, das die MPG unter dem Namen MPG-SFX anbietet, umfasst die Einbindung dieses Dienstes in verschiedene Oberflächen, und zwar in Metalib, darüber hinaus aber z. B. auch in OVID und Web of Science. Diese verschiedenen Web-Angebote fungieren als SFX-Sources. Die über SFX kontextsensitiv verlinkten Dienste werden demgegenüber als SFX-Targets bezeichnet ${ }^{47}$. Es musste jeweils konfiguriert werden, bei welcher Art von Rechercheergebnissen welche Art von Targets angeboten werden; dazu werden sogenannte Thresholds gesetzt, die die Konditionen für die Anzeige der Services festlegen (s. dazu auch Kap. 4.2.3).

\section{Layoutanpassung und Nutzerführung}

Um dem Informationsportal ein zur Max-Planck-Gesellschaft passendes und leicht wiedererkennbares Erscheinungsbild zu geben, wurde das Layout der Metalib-Oberfläche soweit wie möglich an das Layout der Webseiten der Max-Planck-Gesellschaft angepasst. Ein tatsächliches Corporate Design, also ein gemeinsames Erscheinungsbild der (Web-) Angebote wurde erst nach Ablauf der Arbeiten an der Freigabeversion der VLib von der Pressestelle der Max-Planck-Gesellschaft entwickelt und konnte somit nicht mehr rechtzeitig übernommen werden. Für die Version 2.12 von Metalib, die im August 2003 freigegeben wurde, ist das neue Design in Anlehnung an die übrigen Online-Angebote übernommen worden, wobei eine erneute Bearbeitung aller HTML-Seiten erfolgte.

Zur Nutzerführung wurden die mitgelieferten Hilfe- und FAQ-Seiten überarbeitet und mit eigenen Texten versehen. Zusätzlich wurden Feedbackmechanismen eingerichtet.

\section{Benutzerverwaltung}

Neben diesen Konfigurationen musste auch die Benutzerverwaltung eingerichtet und gepflegt werden. Hierzu gehört die Regelung der Nutzerauthentifizierung, die Steuerung der Zugriffsrechte für einzelne Nutzer oder Gruppen von Nutzern sowie die Verwaltung zugelassener IP-Adressen, mit denen von Instituten aus ohne Eingabe der Benutzerkennung zugegriffen werden kann.

\section{Wartung und Systemaktualisierungen}

Bereits in die Implementationsphase fiel ein VersionsUpgrade (von 1.3 auf 2.0) der Metalib- sowie ein Upgrade der SFX-Software. Anschließend erfolgten innerhalb der Metalib-Version mehrere Aktualisierungen. Alle diese Veränderungen wurden zunächst in einer Testumgebung durchgeführt; im August 2003 wurde die Version 2.12 für den Routinebetrieb freigegeben.

Neben den eigentlichen Komponenten der Software für die VLib müssen auch die beteiligten Softwareprodukte gewartet werden und gegebenenfalls, z. B. bei Bekanntwerden von Sicherheitslöchern im Betriebssystem, aktualisiert werden. Dazu gehören neben dem Betriebssystem z. B. der Webserver (hier Apache) und das Datenbanksystem (Oracle), in dem die Informationen zu den eingebundenen Ressourcen verwaltet werden.

Auch die Ressourcenkonfigurationen müssen ständig an neue Gegebenheiten angepasst werden - z. B. bei Softwareupgrades der angeschlossenen Ressourcen oder Änderungen der Hostadressen oder der Lizenzbedingungen. Somit ist eine ständige Überwachung und Überprüfung des Systems erforderlich, um sein Funktionieren und seine Aktualität zu gewährleisten.

\section{Nutzungsstatistiken, Projektdokumentation und Wer- bung}

Statistikfunktionen für die Auswertung der Zugriffszahlen auf das Informationsangebot wurden für SFX bereits mit geliefert; für Metalib konnten zunächst nur die Sitzungen am Webserver gezählt werden, eine differenziertere Statistikfunktion ist erst ab den 2er-Versionen enthalten und muss noch implementiert werden; derzeit laufen Tests. Wichtig ist dabei die Anonymisierung der Protokolldaten aus Datenschutzgründen.

Eine Projektdokumentation wurde erstellt und fortlaufend gepflegt, sie verzeichnet unter anderem die jeweils aktuellen Nutzerzahlen. Werbemaßnahmen, Schulungen und Vorführungen des Systems wurden durchgeführt, um die Bibliothekare und später auch die Wissenschaftler in den Instituten mit der VLib vertraut zu machen. Eine weitere Staffel von Schulungen und Werbemaßnahmen wird Anfang 2004 stattfinden, um die aktuelle VLib-Version mit den enthaltenen neuen Features vorzustellen.

Um einen Austausch mit anderen Nutzern der Produkte Metalib und SFX zu gewährleisten und Einfluss auf die weiteren Entwicklungen durch die Firma zu nehmen, nehmen Vertreter des VLib-Projekts auch an Treffen der nationalen und internationalen User Groups und der Diskussion in deren Mailinglisten teil.

\subsubsection{Freigabe und erste Nutzungserfahrungen}

Zum Zeitpunkt der geplanten Freischaltung der Max Planck Virtual Library am 21.10.2002 waren die meisten Projektziele erreicht worden. Allerdings standen noch einige zum Teil als sehr wichtig erachtete Schritte aus. So konnten z. B. die Ressourcen des Anbieters ISI, die Datenbanken Web of Science (WoS), Web of Knowledge (WoK) und Journal Citation Report (JCR) noch nicht in die Metasuche 
der VLib integriert werden - ein Mangel, der die Attraktivität des Angebots nach Einschätzung der Projektgruppe stark beeinträchtigen dürfte. Die Einbindung dieser Datenbanken sowie weitere Verbesserungen konnten erst mit Version 2 von Metalib realisiert werden.

Diese Version stand zu diesem Zeitpunkt bereits in der Testumgebung zur Verfügung. Allerdings erforderte sienicht zuletzt aufgrund einer grundlegenden Überarbeitung des Layouts, erneut erhebliche Anpassungsarbeiten. Es musste daher entschieden werden, ob die Freigabe in der bisherigen Version 1.3 wie geplant erfolgen oder zunächst die neue Version in Betrieb genommen und das Angebot erst dann den Nutzern zur Verfügung gestellt werden sollte.

Der Vorteil in ersterem Falle hätte in der Einhaltung des Zeitplans und dem nutzbringenden Einsatz der bereits geleisteten Arbeit an der Version 1.3 bestanden; zudem war das Angebot bereits länger angekündigt und seine Freischaltung schon einmal verschoben worden. Nachteilig wäre gewesen, dass die Benutzer zum einen ein Portal kennen lernen würden, dem einige wichtige Komponenten fehlten und dass sie sich zunächst mit einer Suchoberfläche vertraut machen würden, um sich dann in bereits absehbarer Zeit wieder umgewöhnen zu müssen.

Andererseits hätte die Verschiebung der Freigabe bis zum Zeitpunkt der Einsatzbereitschaft von Version 2 eine nicht vertretbare Verzögerung bedeutet, so dass entschieden wurde, zunächst die geplante Freigabe der Version 1.3 durchzuführen, allerdings dabei bereits auf den noch eingeschränkten Funktionsumfang und die geplanten Verbesserungen hinzuweisen - es sollte gewissermaßen eine Freigabe als „Beta-Version“ sein.

In Vorbereitung der Freigabe erfolgte ein vierwöchiger Test durch die Bibliothekarinnen und Bibliothekare; etwa in der Mitte dieses Zeitraumes wurden Schulungen durchgeführt, um die Bibliothekare in die Lage zu versetzen, als Multiplikatoren zu fungieren und den Wissenschaftlern das Angebot näher zu bringen. Begleitend wurden Werbeprospekte entwickelt und verbreitet sowie Informationsveranstaltungen und Vorträge auf verschiedenen Tagungen gehalten.

Erste Nutzungsanalysen nach der Freigabe ergaben, dass das neue Angebot zunächst noch zögernd angenommen wurde und bei unter 100 Zugriffen pro Tag lag, was vor allem auf die noch fehlenden Ressourcen, z. B. Web of Science, sowie die zunächst nur geringe Lokalisierungs- und Personalisierungsmöglichkeit der Angebote zurückgeführt wurde. Dagegen wurde die Komponente SFX, die neben Metalib auch im Rahmen der bereits bekannteren Informationsangebote wie OVID oder STN genutzt werden kann, von Anfang an stark genutzt. Eine Steigerung der Metalib-Nutzung wird erwartet, nachdem bei dem Schulungslauf Anfang 2004 die aktuelle Version mit ihren erweiterten Features einem großen Kreis von Bibliothekaren vorgestellt worden sein wird, die diese Informationen dann an die Wissenschaftler in ihren Instituten weitergeben werden.

\section{Das Serviceangebot der Max Planck Virtual Library}

Der Funktionsumfang der Max Planck VLib aus Sicht der Benutzer umfasst:

- eine Übersicht über alle zentral von der MPG beschafften und (zunehmend) auch über die lokal an den einzel- nen Max-Planck-Instituten verfügbaren elektronischen Informationsressourcen sowie eine Auswahl frei zugänglicher WWW-Quellen,

- eine parallele, verteilte Recherchemöglichkeit in diesen Ressourcen,

- Links zu den URLs der nativen WWW-Oberflächen dieser Ressourcen,

- personalisierte Funktionen (Such-Historie, Alerts, EShelf zur Speicherung von Rechercheergebnissen, individuell abzuspeichernde Ressourcenauswahlliste) sowie

- Verfügbarkeitsrecherchen und zusätzliche Services mit der MPG-SFX-Komponente.

Bereits in Kapitel 3.2 wurden im Zusammenhang mit der Software-Auswahl die Komponenten und Funktionen von Metalib allgemein beschrieben. An dieser Stelle wird auf ihre Implementierung für die Max Planck Virtual Library eingegangen, auf geplante Weiterentwicklungen wird dabei verwiesen ${ }^{48}$

\subsection{Portal und verteilte Suche}

\subsubsection{Ressourcen in VLib}

Die in der Max Planck VLib zusammengestellten Informationsressourcen können auf verschiedene Weise recherchiert und aufgelistet werden: Am linken Fensterrand werden mehrere unterschiedliche Suchmasken angeboten: zunächst eine Auflistung nach Fachgebieten, die weiter nach den Kategorien „Online Catalogs“, „Databases“ und „Journal Collections“ differenziert ist. Eine Zusammenstellung von „New Resources“ informiert über neu eingebundene Quellen. Weitere Suchmöglichkeiten werden mit dem Resource Locator angeboten. Hier kann wahlweise anhand von Ressourcenamen, frei wählbaren Stichworten aus der Beschreibung der Ressource oder jeweils einer Auswahlliste von Schlagworten und Ressource-Typen gesucht werden. Ein „Resource Browser“ ermöglicht das Blättern in einer alphabetischen Liste ausgehend von einem gewählten Einstiegspunkt (s. Abb. 2).

Die Anzeige der gefundenen Informationsressourcen erfolgt in einer Liste. Solche Ressourcen, die für eine Suche unter der VLib-Oberfläche konfiguriert sind („Search“Ressourcen), sind mit einer Checkbox gekennzeichnet; durch Anhaken dieses Kästchens können die gewünschten Quellen markiert und sofort durchsucht werden. Sie können aber auch einer individuellen Ressourcenliste („My Resource List“) hinzugefügt werden. Auf diese Weise kann sich jeder Besucher der Seiten eine individuelle Liste von Informationsquellen zusammenstellen und diese je nach Bedarf verändern. Diese Liste wird für angemeldete Benutzer über die Sitzung hinaus (also auch nach dem Logout) gespeichert; für Benutzer mit Gastzugang bleibt die Liste jeweils nur für die Dauer einer VLibSitzung bestehen.

Geplant ist, künftig unterschiedliche Standard-Benutzerprofile (nach Fachrichtungen oder auch einzelnen MaxPlanck-Instituten) einzurichten und diesen speziell vorkon-

${ }^{48}$ S.a. die Hilfe- und FAQ-Seiten unter <http://vlib.mpg.de>. 


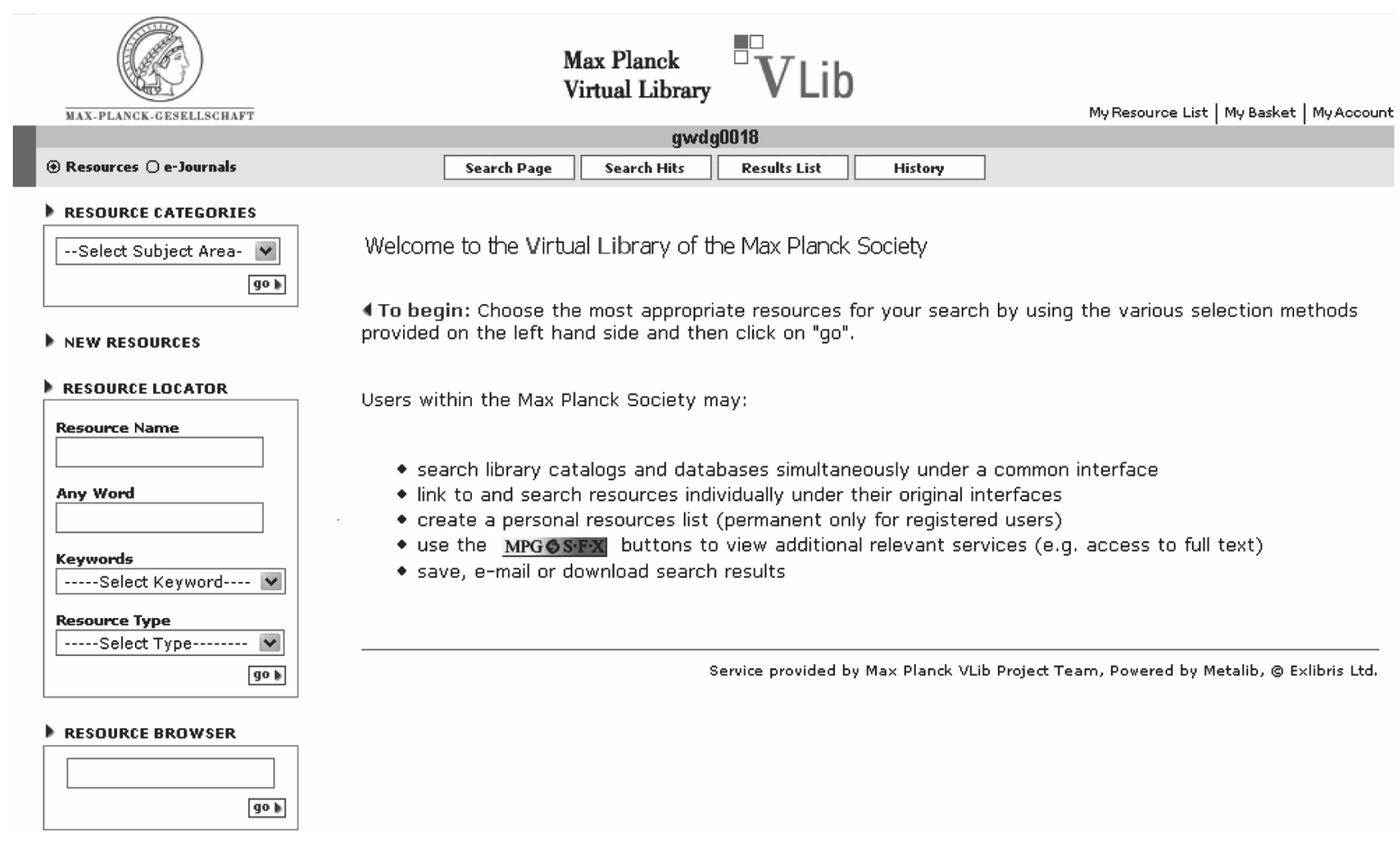

Abb. 2: Einstiegsseite mit Resource-Browser, Max Planck VLib.

figurierte „Default Resource Lists“ anzubieten. So würde Gast- sowie neu eingetragenen Benutzern eine für sie relevante Vorauswahl angeboten, die sie dann jeweils mit ihrer eigenen Auswahl überschreiben können.

Diejenigen Ressourcen, die (noch) nicht für die Suche unter Metalib konfiguriert werden konnten, sind ebenfalls in der Max Planck VLib katalogisiert und mit dem Resource Locator suchbar; für sie steht jedoch nicht die Auswahlmöglichkeit für die VLib-Suche, sondern lediglich ein $\mathrm{Hy}$ perlink zu ihrer nativen Web-Oberfläche zur Verfügung („Link-to"-Ressourcen). Auf diese Weise bietet die Max Planck VLib eine weitgehend vollständige Übersicht über für die Max-Planck-Gesellschaft verfügbare Internetressourcen, selbst wenn diese nicht alle in die VLib-Suche integriert werden können. Die Einbindung von Ressourcen, die nur einem Teil bzw. einzelnen Instituten zur Verfügung stehen, und die Generierung sektions- und institutsspezifischer Sichten ist derzeit in Arbeit.

Mit der aktuellen, seit August 2003 im Einsatz befindlichen Version von Metalib steht zusätzlich ein Modul für elektronische Zeitschriften zur Verfügung. Diese können nach Titel oder ISSN gezielt gesucht werden, es besteht aber auch die Möglichkeit, in einer alphabetischen Liste zu blättern („E-Journals Locator“; s. Abb. 3).

Die von der MPG lizenzierten elektronischen Zeitschriften sind nicht mit der Metalib-Suche recherchierbar; für sie besteht jedoch die Möglichkeit, die MPG-SFX-Funktion zu verwenden, und sie sind als "Link-to"-Ressourcen aus der Max Planck VLib zugänglich. Folgende Ressourcen stehen zur Verfügung:

\section{Bibliothekskataloge}

Derzeit stehen innerhalb der VLib als „Search“- oder „Linkto"-Ressourcen 72 Kataloge von Max-Planck-Institutsbibliotheken (49 davon durchsuchbar) zur Verfügung.

\section{Datenbanken}

In VLib sind 55 Literaturdatenbanken (50 als Search Resources) sowie neun Faktendatenbanken (drei davon als Search Resources) eingebunden. Bei der Einbindung von Datenbanken muss eine Unterstützung durch die jeweiligen Datenbankanbieter gegeben sein, um z. B. die Z39.50-Schnittstelle nutzen zu können. Die entsprechenden Verhandlungen werden in vielen Fällen von der Firma Ex Libris geführt, weil sie die entsprechenden Konfigurationen der Datenbanken in ihrer Knowledge-Base nicht nur einzelnen Kunden, sondern potentiell allen, die die entsprechenden Lizenzen erworben haben, zur Verfügung stellt.

\section{e-Journals}

Von den in der MPG lizenzierten eJournals ist derzeit keines in der VLib als suchbare Ressource enthalten, lediglich die Datenbank Ulrichs Periodicals (OVID) kann unter dieser Oberfläche durchsucht werden. Mit dem e-Journals Browser bzw. dem e-Journals Locator können aber die elektronischen Zeitschriften angezeigt und durch einen Link in der EZB direkt erreicht werden, von wo aus, je nach gegebener Lizenz bzw. bei frei verfügbaren elektronischen Zeitschriften, der Volltext erreicht werden kann. 


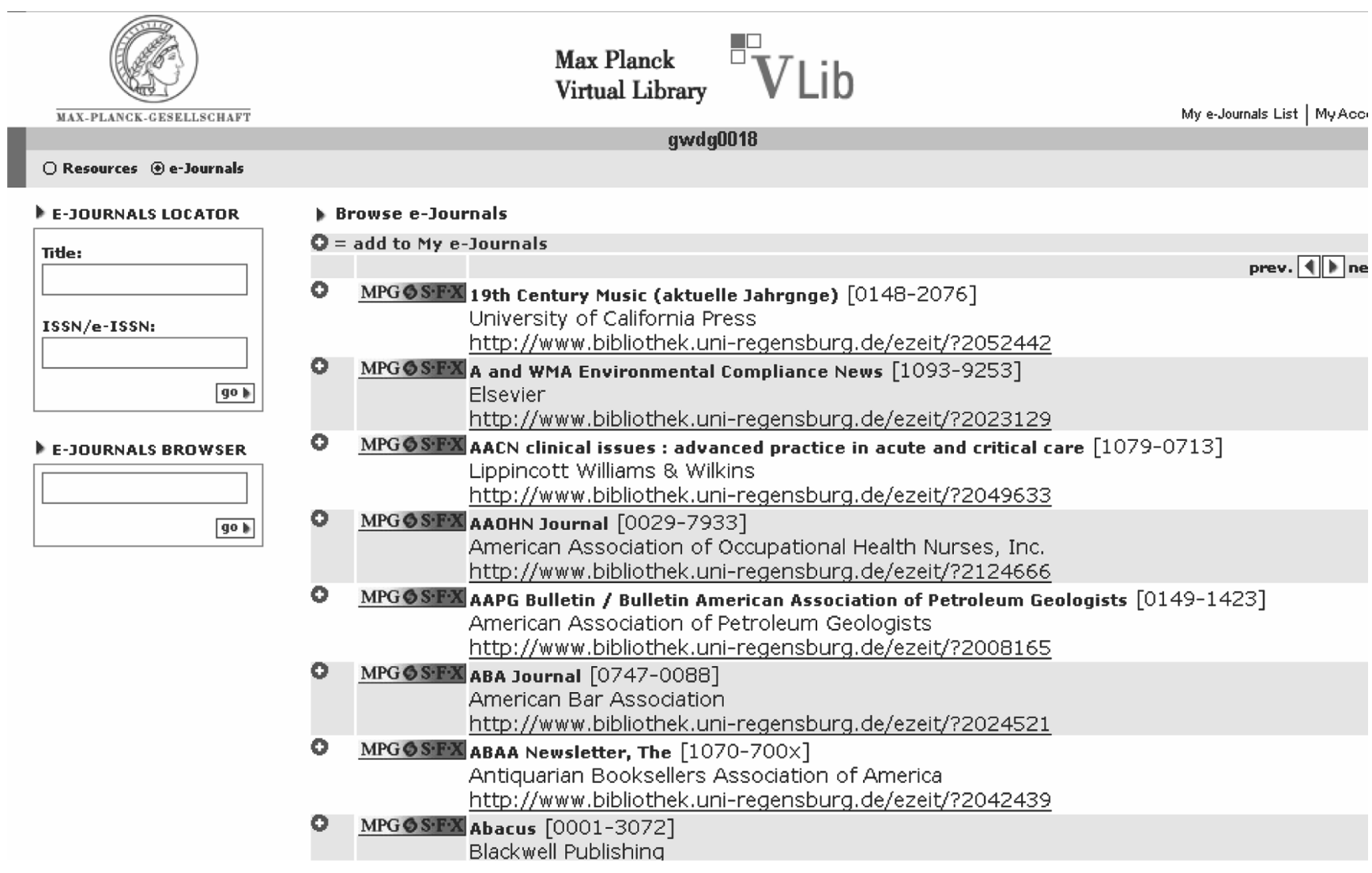

Abb. 3: E-Journals-Locator, Max Planck VLib.

\section{Zeitschriftenverzeichnis}

Im zentralen Verzeichnis der lokalen gedruckten und elektronischen Zeitschriftenbestände sind die Bestände von ca. 35 Max-Planck-Instituten nachgewiesen. Die halbjährlich aktualisierten Datenlieferungen von ZDB und EZB werden in einen Katalog auf dem zentralen Aleph-Server in Göttingen eingespielt ${ }^{49}$.

Aufgrund der Tatsache, dass viele Bibliotheken die Titel ihrer Zeitschriften auch in ihrem Opac verzeichnet haben, können diese Zeitschriften in der VLib an zwei verschiedenen Stellen recherchiert werden bzw. werden bei einer verteilten Suche, bei der sowohl das Zeitschriftenverzeichnis als auch der entsprechende Bibliothekskatalog ausgewählt wurde, doppelt angezeigt. Das wurde in Kauf genommen, denn ein gemeinsames Zeitschriftenverzeichnis wurde als besonders wichtig erachtet, weil eine erhebliche Anzahl von Instituten ihre Zeitschriften bisher überhaupt nicht in einem Online-Katalog verzeichnet hatten, so dass diese Bestände anders überhaupt nicht online recherchierbar wären. Auch durch den seit kurzem verfügbaren e-Journals-Browser (siehe oben) kommen einige Mehrfachnachweise zustande.

\section{Frei verfügbare Web-Ressourcen}

Eine Auswahl frei verfügbarer Online-Ressourcen ist in die Max Planck VLib integriert; diese wird ständig erweitert. Es werden dabei zum einen Vorschläge berücksichtigt, die von Benutzern über ein „Suggest Resource“-Formular (Link auf der Login- und der Logout-Seite) gemacht wurden, zum anderen wurden Bibliothekarinnen und
Bibliothekare aller drei Sektionen der Max-Planck-Gesellschaft aufgefordert, jeweils für ihre Sektion eine Liste relevanter Ressourcen zusammenzustellen. Es wird dabei zunächst insbesondere an die Einbindung von fachbezogenen Portalen (Subject Gateways) gedacht; die Einbindung von frei verfügbaren Webressourcen erfolgt nach einer Qualitätskontrolle.

\subsubsection{Die verteilte Suche}

Bis zu 20 „Search“-Ressourcen können durch Anhaken eines Kästchens für eine gleichzeitige Suche aktiviert werden. Es ist eine Suche über mehrere Begriffe und in folgenden möglichen Feldern möglich: Schlagwort, Titel, Autor, ISBN, ISSN, Jahr (s. Abb. 4).

Ist die Suchanfrage abgeschickt, erscheint ein Fenster, das über den Stand der Suche informiert und sich während des Wartens auf die Antworten der angesprochenen Server mehrmals aktualisiert. Eine Liste der einzelnen angesprochenen Ressourcen gibt den Status der einzelnen Abfragen (z. B. „Searching“) oder gegebenenfalls Fehlermeldungen des angesprochenen Servers wieder; nach Abschluss der Suchanfrage wird hier die Anzahl der erzielten Ergebnissätze für jede Ressource angezeigt. Ferner erscheinen die durchsuchten Ressourcen als Registerkarten, durch Klick auf diese erhält man pro Ressource eine Kurztitelliste der Ergebnisse (s. Abb. 5);

49 <http://aleph.mpg.de/ALEPH/-/start/zdb01>. 


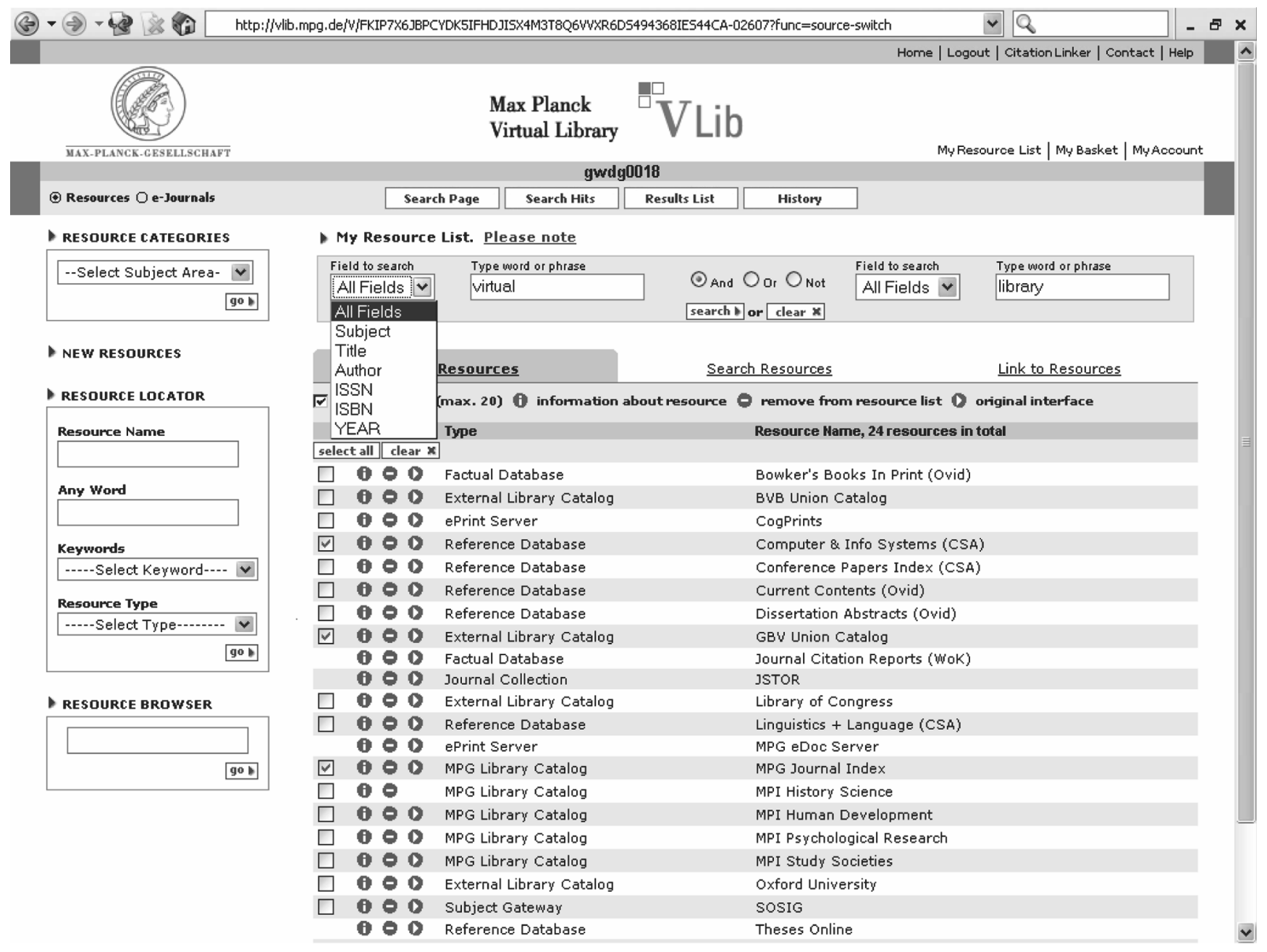

Abb. 4: Suchseite, Max-Planck-VLib.

bei insgesamt bis zu 150 Ergebnissen können diese zusammengeführt und dabei einer Dublettenkontrolle unterzogen werden. Von der Kurztitelliste gelangt man mit Hilfe des „More“-Buttons zur Vollanzeige (s. Abb. 6). Für die Anzeige in der Metalib-Oberfläche werden die von den verschiedenen Servern angefragten Daten aus den verschiedenen bibliothekarischen Datenformaten in ein einheitliches Format (MARC) konvertiert. Mögliche Ausgangsformate sind unter anderem MARC, MAB2, SUTRS, XML oder Dublin Core.

Die Rechercheergebnisse können anschließend im Korb („My Basket“) abgelegt werden, wo sie für eine spätere Weiterverwendung gespeichert werden. Sie können auch in einem von zwei zur Auswahl stehenden Formaten auf den eigenen Rechner heruntergeladen, per Mail verschickt oder aber, durch Export im „Citation Manager“Format, direkt in ein Literaturverwaltungssystem importiert werden (möglich ist dies für EndNote, Reference Manager oder ProCite ${ }^{50}$ ).

In der Suchhistorie werden die bisherigen Suchen gespeichert und können von dort aus erneut abgerufen werden. Weitere Verwendungsmöglichkeiten für die Rechercheergebnisse werden durch den MPG-SFX-Button aktiviert (s. Kap. 4.2).

\subsubsection{Nutzerführung und Feedback-Angebote}

Um den Nutzern der VLib eine effiziente und möglichst einfache Benutzung zu ermöglichen, wurde auf die Anordnung der zur Auswahl angebotenen Ressourcen auf der Suchoberfläche sowie auf die Hilfefunktionen große Sorgfalt verwendet. Eine FAQ-Seite informiert zusätzlich über die Funktionsweise der VLib.

Für Feedback-Äußerungen steht ein Formular zur Verfügung, mit dem eine Nachricht an eine von allen VLib-Administratorinnen und -Administratoren gelesene Mailingliste versandt wird. Je nach Zuständigkeit und Verfügbarkeit antworten dann einzelne Personen auf diese Anfragen und Kommentare; gegebenenfalls werden sie im Administratorenteam diskutiert und fließen in die Planungen zur Weiterentwicklung der VLib ein. Möchte eine Besucherin oder ein Besucher der VLib-Seiten eine noch nicht enthaltene Internetressource für die Integration in die VLib vorschlagen, steht dafür ein spezielles „Suggest Resource“-Formular zur Verfügung.

50 Diese Funktion wird mit Hilfe eines kostenlosen ExportPlugins der Firma ISI ermöglicht (< http://www.isiresearchsoft. com/webcap/rsexport.html>). 


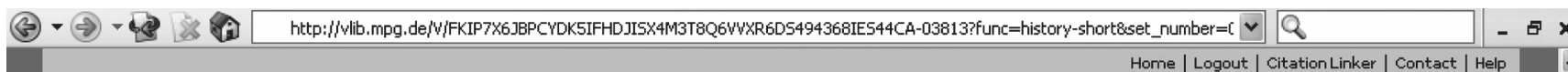

$\begin{array}{ll}\text { Max Planck } & \square \\ \text { Virtual Library } & \text { VL Ib }\end{array}$

MAX-PLANGK-GESELLLBCHAFT

\title{
gwdg0018
}

C Resources $\mathrm{O}$ e-Journals

Search Page

Search Hits

Results List

MýResource List | My Basket | My Account

\author{
- Results List \\ Word from title=(virtual) AND Word from title=(library)
}

Records 1- 20 of 33

Jump to:

View Selected Records | Save Records | Send Records | Add to My Basket | Refine Search | Merge Results

\begin{tabular}{|c|c|c|c|c|c|c|}
\hline \multicolumn{4}{|c|}{ Computer \& Info Systems (CSA) } & MPG Journal Index & \multirow[b]{2}{*}{ Year } & \multirow[b]{2}{*}{ Resource } \\
\hline$\#$ & & & Author & Title & & \\
\hline 1 & more & $\square$ & Hanson, Ardis & Building a virtual library & 2003 & GBV Union Catalog MPG S S.F.X \\
\hline 2 & more & $\square$ & & LANIC :Latin American Network Information Center & 2001 & GBV Union Catalon MPG S S.F.X \\
\hline 3 & more & $\square$ & & $\begin{array}{l}\text { Building the virtual reference desk in a } 24 / 7 \text { world : Library of Congress and OCLC } \\
\text { symposium at ALA Midwinter } 2001 \text {, January } 12,2001 \text {, Washington, D.C. }\end{array}$ & 2001 & GBV Union Catalog MPG S S.F.X \\
\hline 4 & more & $\square$ & $\begin{array}{l}\text { Kemp, Thomas } \\
\text { Jay }\end{array}$ & The genealogist's virtual library :full-text books on the world wide web & 2000 & GBV Union Catalon MPG S S:F:X] \\
\hline 5 & more & $\square$ & & Virtual Library of Anglo-American Culture - Literature & 2000 & GBV Union Catalon MPG S S.F.X \\
\hline 6 & more & $\square$ & & Virtual Library of Anglo-American Culture - History & 2000 & GBV Union Catalon MPG SS·F:X \\
\hline 7 & more & $\square$ & $\begin{array}{l}\text { Stielow, } \\
\text { Frederick }\end{array}$ & Creating a virtual library : a how-to-do-it manual for librarians & 1999 & GBV Union Catalon MPG @S·F-X \\
\hline $\mathbf{8}$ & more & $\square$ & $\begin{array}{l}\text { Saunders, } \\
\text { Laverna M. }\end{array}$ & The evolving virtual library & 1999 & GBV Union Catalog MPG S S.F:X \\
\hline 9 & more & $\square$ & $\begin{array}{l}\text { LaGuardia, } \\
\text { Cheryl }\end{array}$ & Recreating the academic library :breaking virtual ground & 1998 & GBV Union Catalog MPG S S.F:X] \\
\hline 10 & more & $\square$ & & Biblioteca virtual dos autores portugueses :Virtual library of Portuguese authors & 1998 & GBV Union Catalon MPG SS.F.X \\
\hline 11 & more & $\square$ & $\begin{array}{l}\text { Saunders, } \\
\text { Laverna M. }\end{array}$ & The evolving virtual library : visions and case studies & 1998 & GBV Union Catalog MPG SS.F:X \\
\hline 12 & more & $\square$ & & The virtual law library :personal edition & 1998 & GBV Union Catalog MPG QS.F.X \\
\hline 13 & more & $\square$ & $\begin{array}{l}\text { Saunders, } \\
\text { Laverna M. }\end{array}$ & The evolving virtual library : visions and case studies & 1998 & GBV Union Catalag MPG SS.F:X \\
\hline 14 & more & $\square$ & Pastine, & Collection development : access in the virtual library & 1997 & GBV Union Catalog MPG S S.F:X \\
\hline
\end{tabular}

Abb. 5: Ergebnisliste, Max Planck Vlib.

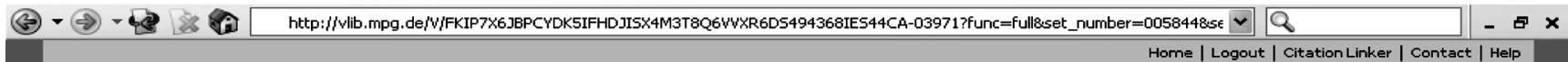

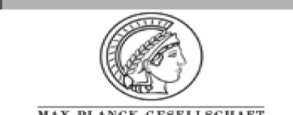

MAX-PLANGK-GESELLLSCHAFT

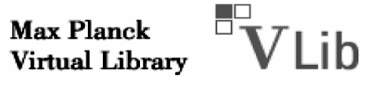

(9) Resources $O$ e-Journals

gwadg0018

Mỵ Resource List | My Basket | MỵAccount

- Full Yiew

Word from title =(virtual) AND Word from title=(library); Record 7 of 33

Choose format: Standard original Converted

\begin{tabular}{|c|c|}
\hline & Add to Basket $\square$ Save $D$ Sent to ${ }^{-1}$ MPG S S.F.X \\
\hline Resource & - GBV Union Catalog \\
\hline Title & Creating a virtual library : a how-to-do-it manual for librarians / ed. by Frederick Stielow. \\
\hline Add.Author/Editor & $\diamond$ Stielow, Frederick, 1946- \\
\hline YEAR & 1999 \\
\hline \multirow[t]{5}{*}{ Subject } & - Library information networks \\
\hline & - Web sites Design \\
\hline & - Libraries Special collections Computer network resources \\
\hline & - Diqital libraries Desiqn \\
\hline & - Elektronische Bibliothek \\
\hline ISBN & 1555703461 \\
\hline Note & Webliography S. [171] - 177 . \\
\hline LC no. & Z674.75.W67 \\
\hline Dewey no. & 025.002855276 \\
\hline Imprint & New York [u.a.] : Neal-Schuman , 1999.. \\
\hline Description & XI, $184 \mathrm{~S}$ Ill. \\
\hline Series & How-to-do-it manuals for librarians, 91 \\
\hline \multirow[t]{2}{*}{ Holdings } & * GBV Union Cataloq \\
\hline & 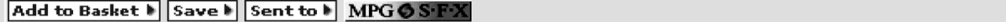 \\
\hline
\end{tabular}

Abb. 6: Vollanzeige eines Suchergebnisses, Max Planck VLib. 


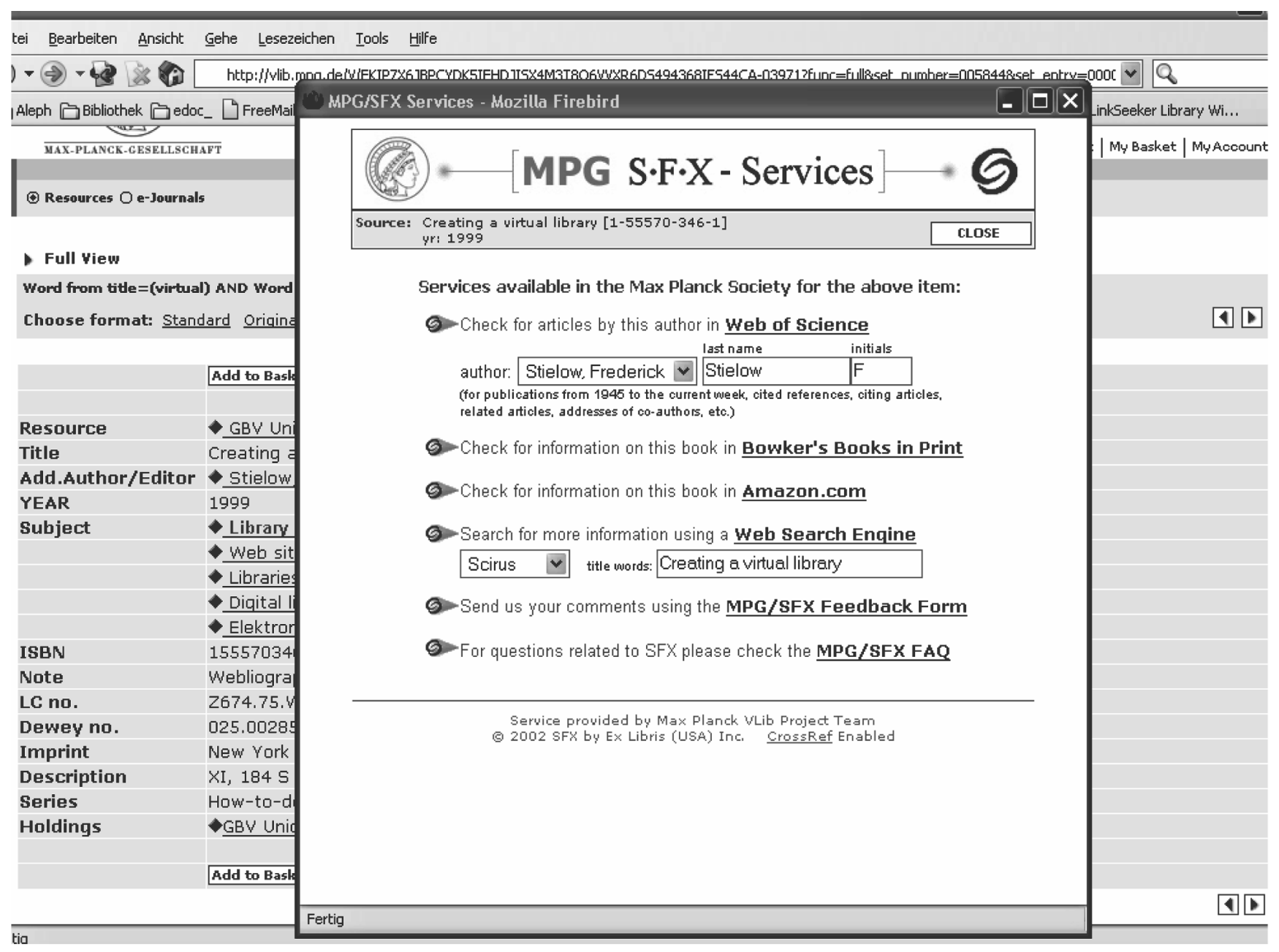

Abb. 7: MPG-SFX-Fenster zum Ergebnissatz aus Max Planck VLib-Recherche.

Die Unterstützung der Nutzer und die Kommunikation mit innen werden als äußerst wichtige Komponenten des VLibAngebots gesehen, die für die Akzeptanz dieses Portals eine große Rolle spielen.

\subsection{MPG-SFX}

Ein weiterer zentraler Service der Max Planck VLib ist das Angebot der kontextsensitiven Verlinkung von Ergebnissätzen bzw. deren Bestandteilen aus zu anderen relevanten Services. Dieser Dienst wird mit dem Programm SFX (ursprüngliche Bezeichnung „Special Effects“, jetzt „Context Sensitive Reference Linking ") realisiert, das von Herbert van de Sompel entwickelt wurde ${ }^{51}$. Die Funktionsweise von SFX basiert auf dem Interoperabilitäts-Protokoll OpenURL, das den Austausch von Metadaten zwischen verschiedenen Informationsressourcen ermöglicht ${ }^{52}$.

Die SFX-Software wurde zusammen mit Metalib erworben, kann aber nicht nur in dieses Portal, sondern auch in andere Suchoberflächen eingebunden werden, sofern deren Anbieter dies ermöglicht haben. Die Ressourcen, von denen aus SFX genutzt werden kann, werden SFXSources genannt.

Neben dem in einer dieser Suchoberflächen gewonnenen Rechercheergebnis, teils auch neben einzelnen Feldern (Autor, Titel, Journal usw.) erscheint ein Button, der per Mausklick zu einem Fenster mit einem kontextbezo- genen Auswahlmenü zusätzlicher Services führt. Dazu zählen z. B. die Weiterleitung eines Begriffs aus dem Ergebnisdatensatz an eine Web-Suchmaschine, die Weiterleitung eines gefundenen Katalogisats an einen Bibliothekskatalog, die Suchen nach einer Monografie in einem Buchhandelsverzeichnis oder aber der Link zum Volltext eines Artikels in einem lizenzierten e-Journal. Alle Systeme, deren Services über das SFX-Menü verlinkt sind, werden als SFX-Targets bezeichnet ${ }^{53}$ (s. Abb. 7).

51 <http://www.sfxit.com>; zur Entwicklung s. Van de Sompel, Herbert und Patrick Hochstenbach (1999). Reference linking in a hybrid library environment. Part 1: frameworks for linking. D-Lib Magazine 5, no. 4 <http://www.dlib.org/dlib/april99/ van_de_sompel/04van_de_sompel-pt1.html>; Part 2: SFX, a generic linking solution. D-Lib Magazine 5, no. $4<\mathrm{http}$ ://www. dlib.org/dlib/april99/van_de_sompel/04van_de_sompel-pt2. html>; Part 3: Generalizing the SFX solution in the "SFX@Ghent \& SFX@LANL" experiment. D-Lib Magazine 5, no. 10 <http://www.dlib.org/dlib/october99/van_de_sompel/ 10van_de_sompel.html>.

52 Mittlerweile ein NISO-Standard, s. <http://www.niso.org/ committees/committee_ax.html>. Informationen zu OpenURL auch auf der SFX-Website <http://www.sfxit.com>.

${ }^{53}$ Eine allgemeine Liste von SFX-Sources (nicht MPG-spezifisch) s. unter <http://www.sfxit.com/sources-list.html>; mögliche Targets s. unter <http://www.sfxit.com/targets.html>. 


\section{(9) $[$ MPG - CitationLinker $] \longrightarrow$ S S.F.X}

The citation Linker will point you to specific MPG/SFX Services that are available for the reference you have indicated, including links to the full text or to relevant database entries. Enter as much information as you have in the appropriate form and click the MPG SFX button below.

\begin{tabular}{|l|}
\hline CLOSE \\
\hline CLEAR \\
\hline
\end{tabular}

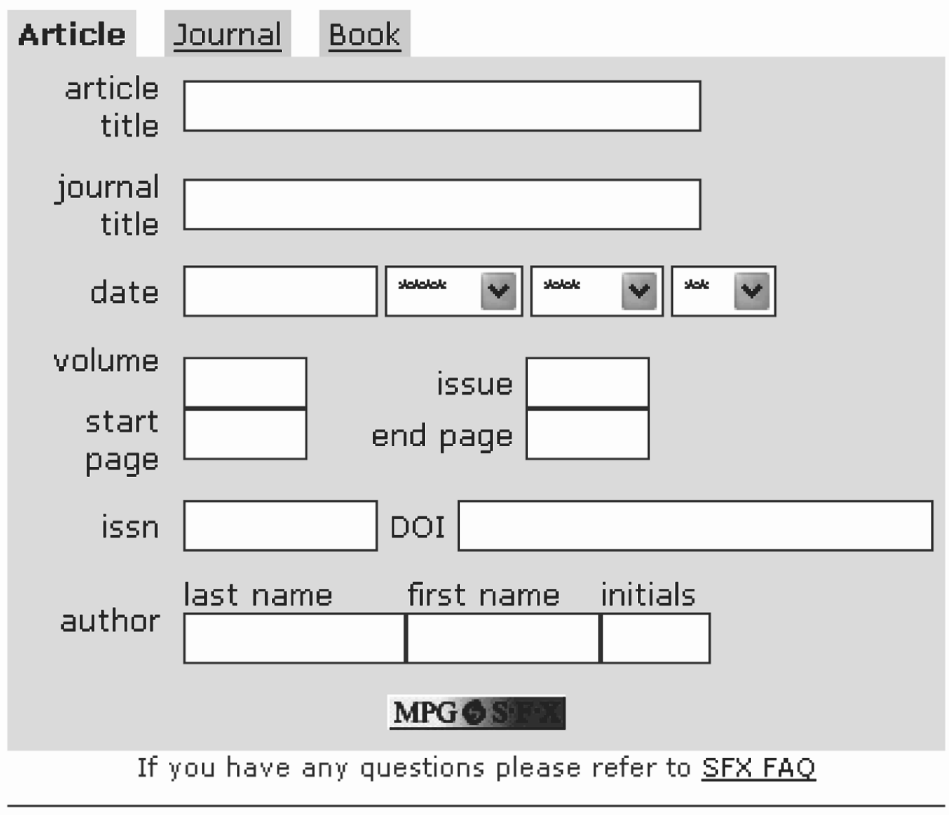

Service provided by Max Planck VLib Project Team

Abb. 8: MPG Citation Linker.

\subsubsection{MPG-SFX-Sources}

Als MPG-SFX-Sources wurden außer Metalib auch OVID, CSA und das Web of Science (WoS), das MPG-Zeitschriftenverzeichnis sowie der eDoc-Server der Max-PlanckGesellschaft konfiguriert. Auch die meisten Aleph-Bibliothekskataloge von Max-Planck-Instituten fungieren als MPG-SFX-Sources; zudem wird angestrebt, diese Funktion für weitere Informationsressourcen, unter anderem Science Direct, JSTOR und Faculty of 1 000, zu implementieren.

\subsubsection{Max Planck Citation Linker}

Der MPG-SFX-Dienst kann auch unabhängig von der Nutzung einer SFX-fähigen Suchoberfläche genutzt werden: Über den Max Planck Citation Linker ${ }^{54}$ kann eine bibliografische Angabe direkt eingegeben und SFX dann von dort aus aktiviert werden (s. Abb. 8).

\subsubsection{MPG-SFX-Targets}

Nach Analyse der bibliografischen Angaben werden im SFX-Fenster verschiedene Links angeboten. Welche das sind, wird durch die Einrichtung von „Thresholds" definiert, in denen die Bedingungen festgelegt sind, aufgrund derer bestimmte Dienste für einen Datensatz oder Bestandteil eines solchen angezeigt werden oder nicht. So erscheint es wenig sinnvoll, zu einem Datensatz etwa eine Anfrage nach elektronischer Verfügbarkeit an die EZB anzubieten, wenn der Benutzer auch bereits direkt aus dem SFX-Menü heraus zum Volltext gelangen kann.

Ein neues Feature bei SFX ist die Möglichkeit der Verwendung von IP-Adressgruppen als Thresholds, so dass die Targets, z. B. Bibliothekskataloge, auch je nach Institut eingebunden werden können. Das wird die Einrichtung auch solcher Dienste ermöglichen, die nicht allen Instituten zur Verfügung stehen und ist ein wesentlicher Bestandteil der geplanten Lokalisierungen des VLib-Angebots. Folgende Arten von Targets sind derzeit eingerichtet und werden je nach Kontext angeboten: 


\section{Volltexte und Bestandsangaben bei e-Journals}

Bei Artikeln aus elektronischen Zeitschriften wird der direkte Zugang zum Volltext angeboten, sofern eine MPGweite Lizenz für die jeweilige Zeitschrift vorliegt oder diese frei zugänglich ist. Bei den Zeitschriften einiger Verlage konnte zunächst trotz vorhandener Lizenz allerdings nicht direkt zum Volltext, sondern nur auf die Ebene der Startseite der Zeitschrift verlinkt werden. Um auch für diese Zeitschriften eine direkte Durchschaltung zum Volltext zu ermöglichen, wurde eine Mitgliedschaft bei CrossRef eingegangen. CrossRef ist eine Initiative, die unter Nutzung des Digital Object Identifiers (DOI) die Verlinkung von wissenschaftlichen Informationsressourcen verschiedener Provenienz untereinander unterstützt. So konnte bereits für eine Reihe von Zeitschriften der Direktzugriff auf Artikelebene realisiert werden.

\section{Nachweise in Bibliothekskatalogen}

Es wurden zunächst exemplarisch einzelne Kataloge von Max-Planck-Institutsbibliotheken als SFX-Targets eingebunden. Erst künftig, wenn SFX für einzelne Institute spezifisch eingerichtet werden kann, ist die Einbindung weiterer Bibliothekskataloge geplant, denn es wird als wenig sinnvoll angesehen, über SFX die Bestände zu einem Titel in einer zu großen Auswahl von Bibliotheken gleichzeitig anzubieten.

\section{Dokumentenbestellung}

Erste Gespräche mit Subito zur Einbindung dieses Dokumentenlieferdienstes in das SFX-Angebot der MPG sind erfolgt.

\section{Datenbanken}

Für die lizenzierten Datenbanken wurden verschiedene Dienste eingerichtet, z. B. für das Web of Science eine Autorensuche („Search this author in WoS“) oder für die unter OVID verfügbare Datenbank Current Contents eine Suche nach dem Inhaltsverzeichnis einer gefundenen Quelle („Find ToC in CC“). In PubMed oder den Dissertation Abstracts kann nach einem zitierten Titelsatz recherchiert werden (,getCitedRecord“-Funktion).

\section{Suchmaschinen}

Außerdem wurde eine Suche nach bestimmten Inhalten des Rechercheergebnisses in einigen Suchmaschinen eingerichtet ${ }^{55}$.

\section{Nachschlagewerke}

Als Online-Nachschlagewerke hat die MPG die Oxford Reference Works lizenziert. Diese können wahrscheinlich ebenfalls bald als SFX-Targets eingebunden werden.

\subsection{Zugriffsmöglichkeiten und Nutzeradministra- tion}

Die Max Planck VLib ist mit einem Gastzugang öffentlich zugänglich, bietet den Zugang zur Gesamtheit der hier nachgewiesenen Ressourcen, weil viele von ihnen kostenpflichtig sind, aber nur angemeldeten Benutzern. Die Anmeldung erfolgt mittels eines Antragsformulars, auf dem Interessierte eine Benutzerkennung beantragen können; bei der Bearbeitung dieses Antrags wird ihre Zugehörigkeit zur Max-Planck-Gesellschaft und damit ihre Berechtigung für die Nutzung eines Zugangs verifiziert.

Neben der persönlichen Authentifizierung werden die Zugriffsberechtigungen auch anhand einer Überprüfung der IP-Adresse des Rechners gesteuert, von dem aus die VLib genutzt wird. Es ist daher von einem Arbeitsplatz innerhalb des IP-Adressbereichs der MPG möglich, auch mit dem Gastzugang auf alle für die MPG lizenzierten Quellen zuzugreifen; in diesem Falle entfällt jedoch die Möglichkeit, persönliche Einstellungen über die Sitzung hinaus zu speichern.

Künftig wird außerdem ein IP-Tunneling eingerichtet. Das bedeutet, dass Benutzer, auch wenn sie nicht von einer berechtigten IP-Adresse aus auf VLib zugreifen, die angeschlossenen Server mit IP-beschränktem Zugang werden erreichen können.

\section{Schlussbetrachtungen}

Die vorangegangene Darstellung der Max Planck Virtual Library, ihrer Entstehungsgeschichte und ihrer Rolle in der Informationsinfrastruktur der Max-Planck-Gesellschaft hat die Komplexität eines solchen Projekts und die Interdependenz der miteinander verknüpften Informationssysteme gezeigt. Um ein qualitativ hochwertiges und für die Nutzer gewinnbringendes webbasiertes Zugangssystem zu Online-Quellen zu bieten, müssen viele einzelne Schwierigkeiten überwunden oder durch "Workaround“ elegant umgangen werden.

\section{One-Stop-Shopping mit Hindernissen}

Im Kontext eines solchen Meta-Informationssystems entstehen Probleme besonders im Zusammenhang mit den Schnittstellen zwischen verschiedenen Softwaresystemen sowie mit den Protokollen und Formaten, die die Übertragung und Konversion bibliografischer Daten regeln. Auch die Übertragung zwischen verschiedenen Zeichensätzen kann, bei der Darstellung von Sonderzeichen, eine Fehlerquelle sein. Viel akribische Kleinarbeit ist vonnöten, um eine Virtuelle Bibliothek funktionstüchtig zu machen und zu erhalten. Nicht alle auf der VLib-Oberfläche angebotenen Funktionen werden beispielsweise von allen lokalen Systemen unterstützt, wie z. B. die beliebig wählbare Sortierung der Ergebnissätze. Änderungen in den einzelnen angeschlossenen Systemen erfordern Anpassungen der Konfigurationen - und somit ständige Wachsamkeit seitens der Administratoren.

\section{Für und Wider integrierter Zugangssysteme}

Angesichts des Aufwandes, der bei Errichtung und Pflege eines solchen Systems entsteht, erhebt sich die Frage nach dem Sinn und Zweck eines solchen Angebots.

55 Alta Vista, dmoz, Google, Scirus, Teoma und Yahoo. 
Kritiker integrierter Zugangssysteme heben immer wieder hervor, dass jede gemeinsame Suchoberfläche, obgleich (oder vielmehr gerade weil) sie dem bibliothekarisch nicht vorbelasteten Benutzer einen bequemen, intuitiv erlernbaren Zugang zu relevanter wissenschaftlicher Information suggeriere, zu Fehleinschätzungen führen könne.

Dies wird damit begründet, dass Suchoberflächen, die heterogene Systeme gemeinsam abfragen, immer notgedrungen mit dem kleinsten gemeinsamen Nenner an Suchmöglichkeiten arbeiten müssen und ihren Nutzern somit niemals die fein granulierten Abfragemöglichkeiten erlauben, die in den Oberflächen der einzelnen angeschlossenen Systeme angeboten werden. Auch wird die Fehleranfälligkeit der Recherchen betont - wenn z. B. in einer gemeinsamen Suche nach einer Jahreszahl gefragt wird, die in einem der durchsuchten Kataloge schlicht nicht erfasst ist, erhält die oder der Suchende null Ergebnisse zurück geliefert - obwohl es durchaus möglich ist, dass der relevante Datensatz sich in der befragten Quelle befindet.

Sollten den Wissenschaftlern also weiterhin eine Vielzahl an Datenquellen mit unterschiedlicher Suchlogik, die vermutlich nur von Informationsspezialisten zu durchschauen ist, ohne vereinheitlichenden Zugang zugemutet werden? Bleibt das One-Stop-Shopping, der „bruchlose Informationsraum" innerhalb des WWW ein unerreichbares Ideal?

Wenn die Einwände gegen solche Systeme sicherlich zutreffend sind, ist doch ihre generelle Zweckmäßigkeit nicht von der Hand zu weisen. Die Nachteile der Unschärfe von Meta-Suchen und der gelegentlichen Fehler werden dadurch aufgewogen, dass die Benutzer durch integrierte Zugangssysteme, sei es für ihr Fachgebiet oder die Institution, der sie angehören, eine Übersicht über die dort verfügbaren Ressourcen erhalten und möglicherweise durch ein solches System auf Quellen aufmerksam werden, von deren Existenz sie zuvor nichts wussten. Nicht zuletzt dienen solche Portale somit auch der Sichtbarkeit - nach innen und außen - des Bestandes an Arbeitsmitteln der Institution oder Organisation, für die sie eingerichtet wurden und erfüllen so eine wichtige Funktion in deren Öffentlichkeitsarbeit.

Die zwangsläufig flachen Suchmöglichkeiten von Metakatalogen sowie die Fehleranfälligkeit können nicht geleugnet werden - allerdings kommen fehlerhafte Resultate auch bei der - uneingeweihten - Suche in einzelnen Datenbanken oder Katalogen vor. Die Suchanfragen in einem Metakatalog sollten in der Regel eher einem ersten Überblick über die Ressourcen und deren Inhalte dienen; für detailliertere Anfragen werden sich die Benutzer mit großer Wahrscheinlichkeit ohnehin den originären Systemen zuwenden oder einen Informationsspezialisten kontaktieren (weshalb der Einbau einer „Ask a Librarian"-Maske in ein solches Portal sinnvoll wäre - immerhin findet sich bereits an vielen Stellen in den Hilfetexten zur VLib der Verweis auf die Fachkräfte in den lokalen Bibliotheken).

\section{Fazit}

Aus den Erfahrungen mit der Virtuellen Bibliothek der MaxPlanck-Gesellschaft ist vor allem zu lernen, dass ein integriertes Zugangssystem in einem fachlich heterogenen und geografisch verteilten Umfeld an Attraktivität für seine potentiellen Benutzer - Bibliothekare wie auch Wissenschaftler - zu wünschen übrig lässt, solange keine ausreichende Differenzierung des Angebots nach fachlichen sowie lokalen Gegebenheiten vorhanden ist.

Als von Beginn an sehr erfolgreich haben sich hingegen die MPG-SFX-Services erwiesen. Daran ist zu ersehen, dass der Ansatz, die Integration und Interoperabilität der verschiedenen Datenquellen zu fördern, richtig ist und weiter vorangetrieben werden muss.

Die Bemühungen zur Weiterentwicklung des Portals zielen folgerichtig - neben einer Ausweitung des Angebots an Ressourcen und einer stetigen Verbesserung ihrer Verlinkung untereinander - auf "maximale Personalisierung ${ }^{456}$ des Portals ab. Somit kann erwartet werden, dass die Bekanntheit und Akzeptanz der VLib mit ihrem weiteren Reifeprozess zunehmen wird.

\section{Anschrift der Autorin:}

Anke Bruns

GWDG

Am Fassberg

D-37077 Göttingen

E-Mail: anke.bruns@gwdg.de 